\title{
The exact solution of the Riemann problem in relativistic magnetohydrodynamics
}

\author{
By BRUNO GIACOMAZZO ${ }^{1}$ AND LUCIANO REZZOLLA ${ }^{1,2}$ \\ ${ }^{1}$ SISSA, International School for Advanced Studies and INFN, Trieste, Italy \\ ${ }^{2}$ Department of Physics, Louisiana State University, Baton Rouge, USA
}

(Received 15 July 2005 and in revised form 26 January 2006)

We discuss the procedure for the exact solution of the Riemann problem in special relativistic magnetohydrodynamics (MHD). We consider both initial states leading to a set of only three waves analogous to the ones in relativistic hydrodynamics, as well as generic initial states leading to the full set of seven MHD waves. Because of its generality, the solution presented here could serve as an important test for those numerical codes solving the MHD equations in relativistic regimes $\dagger$.

\section{Introduction}

As first formulated by Riemann more than a hundred years ago, the solution of the onedimensional Riemann problem in hydrodynamics consists of determining the temporal evolution of a fluid which, at some initial time, has two adjacent uniform states characterized by different values of uniform velocity, pressure and density. These initial conditions completely determine the way in which the discontinuity will decay after removal of the barrier separating the initial "left" and "right" states.

The Riemann problem has ceased to be merely academic and has gained enormous importance when it was realized that its numerical solution can serve as the building block of hydrodynamical codes based on Godunov-type finite difference methods (Godunov 1959). In such methods, the computational domain is discretized and each interface between two adjacent grid-zones is used to construct the initial left and right states of a "local" Riemann problem. The evolution of the hydrodynamical equations is then obtained through the solution across the computational grid of the sequence of local Riemann problems set up at the interfaces between successive grid-zones (see Godunov 1959, but also Martí \& Müller 2003 and Font 2003 for the use of the Riemann problem in relativistic regimes).

In general, the Riemann problem requires the solution of a nonlinear algebraic system of equations written as a function of a single unknown quantity (e.g. the total pressure at the contact discontinuity in purely hydrodynamical problems). With the exception of few trivial initial configurations, the solution of the Riemann problem cannot be obtained analytically but requires a numerical approach. The solution found in this way is referred to as the "exact" solution of the Riemann problem, to distinguish it from the "approximate" solution of the Riemann problem, which is instead obtained when the system of equations is reduced to a locally-linear form (an exhaustive discussion of approximate Riemann solvers can be found in Toro 1999). It is therefore useful to stress that although named "exact", the solution of the Riemann problem is necessarily obtained with a small but nonzero truncation error.

The exact solution of the Riemann problem in relativistic hydrodynamics has been obtained

$\dagger$ The numerical code computing the exact solution is available from the authors upon request. 
only rather recently and was proposed by Martí \& Müller (1994) for flows that are purely along the direction normal to the initial discontinuity. This work has then been extended to the case in which tangential velocities are present (Pons et al. 2000) and improved in efficiency by exploiting the relativistic invariant relative velocity between the two states to predict the wave pattern produced (Rezzolla \& Zanotti 2001 and Rezzolla et al. 2003). The relevance of these calculations has not been restricted to fundamental issues of relativistic hydrodynamics. Quite the opposite, these solutions have been of great importance for the testing of complex multidimensional codes implementing High Resolution Shock Capturing (HRSC) methods, and that are based on the approximate or exact solution of Riemann problems at the interfaces between the numerical cells (LeVeque 1992). These codes have then been used in various simulations in either fixed (Aloy et al. 1999, Font \& Daigne 2002, Zanotti et al. 2003) or dynamical spacetimes (Duez et al. 2004, Shibata \& Sekiguchi 2005|Baiotti et al. 2005).

This intense and recent development of numerical codes for the solution of the relativistic hydrodynamic equations has been accompanied by an equally intense development of codes solving the equations of magnetohydrodynamics (MHD) in relativistic regimes. The reason behind this activity is the widespread expectation that strong magnetic fields are crucial in the study and explanation of several puzzling astrophysical phenomena such as relativistic jets or $\gamma$-ray bursts. As a result, and in the hope of clarifying issues in relativistic astrophysics which cannot be described satisfactorily through analytic techniques, several groups have recently constructed numerical codes solving the equations of relativistic MHD on either fixed spacetimes (see, for example, Del Zanna et al. 2003 and Komissarov 1999 for a flat background and Gammie et al. 2003. De Villiers et al. 2003, Komissarov 2004 Mizuno et al. 2004, Fragile 2005 Antón et al. 2005 for a black-hole background) or in fully dynamical spacetimes (Duez et al. 2005).

Just like their hydrodynamical counterparts, some of these codes are based on the solution of a local Riemann problem suitably formulated for a magnetized fluid, and all are meant to be used for ultrarelativistic flows. However, unlike their hydrodynamical counterparts, these codes cannot benefit from the comparison with the exact solution of the Riemann problem in relativistic MHD. The literature on the Riemann problem in MHD is, in fact, much more limited and a general exact solution was found rather recently and for a Newtonian fluid only (Ryu \& Jones 1995, Falle et al. 1998). The background knowledge in this area is even more scarce for a relativistic fluid and while no general exact solution has been proposed yet, recent work has been made to derive an exact solution in the particular case in which the magnetic field of the initial states is tangential to the discontinuity and orthogonal to the fluid velocity (Romero et al. 2005). Besides having a larger set of equations when compared to the corresponding problem in relativistic hydrodynamics, a considerable addition to the complexity of the Riemann problem in relativistic MHD is represented by the fact that the mathematical structure of the problem itself is modified and the system of equations is no longer strictly hyperbolic (Lichnerowicz 1967) $\dagger$. The possibility of having coincident eigenvalues poses the question of the uniqueness of the solutions and this represents then a problem within the problem. As we will comment also later on, a lively debate on these issues is presently ongoing and progress is starting to be made, although first results are known in Newtonian MHD only (see Torrilhon 2003b). Because the focus of this work is the exact solution of the Riemann problem in relativistic MHD as an aid to the development of numerical codes, hereafter we will adopt the working assumption that the Riemann problems considered here have a solution and that this solution is unique. Clearly, this hypothesis avoids the issue rather than solving it, but it allows for

$\dagger$ We recall that a systems of $m$ quasi-linear partial differential equations is said to be hyperbolic if the matrix of coefficients has $m$ real eigenvalues; furthermore, the system is said to be totally or strictly hyperbolic if the eigenvalues are real and also all distinct. 
a marked progress at least in those cases in which compound waves are not found in numerically approximate solutions.

A direct and important consequence of the scarcity of works in this area of fundamental relativistic MHD is that the validation of modern complex MHD codes for the most elementary and yet demanding tests has not been made in a quantitative manner for generic initial conditions. Rather, it has taken place through the qualitative comparison with the large set of test-problems in relativistic MHD meticulously collected over the years (see, for instance, Komissarov 1999 and Balsara 2001). It should be recognized, however, that for non-generic initial states it is sufficient to have exact solutions for MHD shocks and rarefactions as this covers all types of basic hyperbolic waves of the system and indeed exact solutions of this type were used by Komissarov (1999) for quantitative testing.

On the other hand, the purpose of this paper is to present the procedure for the exact solution of the Riemann problem in relativistic MHD with generic initial conditions. Our approach considers both initial states with a zero component of the magnetic field along the flow and leading to a set of only three waves analogous to the ones in relativistic hydrodynamics, as well as generic initial states leading to the full set of seven MHD waves. The approach discussed for the numerical solution is based on a "hybrid" approach which adopts different sets of equations according to the values of the normal magnetic field and that has turned out to be crucial for a successful solution.

The paper is organized as follows: Section 2] contains the basic equations of relativistic MHD, while Section 3 describes the strategy used to solve the Riemann problem numerically and which combines the methods discussed in Section 4 and Section 5 Section 6 focuses on the details of the numerical implementation and discusses the solution of a number of tests that have become standard references. Finally, the conclusions are collected in Section 7

We use a spacelike signature $(-,+,+,+)$ and a system of units in which $c=1$. Greek indices are taken to run from 0 to 3, Latin indices from 1 to 3 and we adopt the standard convention for the summation over repeated indices. Finally we indicate 3 -vectors with an arrow and use bold letters to denote 4-vectors and tensors.

\section{Equations of Relativistic MHD}

Consider an ideal but magnetized relativistic fluid with an energy-momentum tensor given by

$$
T^{\mu \nu}=\left(\rho+\rho \epsilon+p_{\mathrm{g}}+2 p_{\mathrm{m}}\right) u^{\mu} u^{\nu}+\left(p_{\mathrm{g}}+p_{\mathrm{m}}\right) \eta^{\mu \nu}-b^{\mu} b^{\nu},
$$

where $\rho$ is the rest mass density, $\epsilon$ the specific internal energy, $p_{\mathrm{g}}$ the gas pressure, $p_{\mathrm{m}}$ the magnetic pressure, $u^{\mu} \equiv W\left(1, v^{x}, v^{y}, v^{z}\right)$ the four-velocity, $W \equiv 1 / \sqrt{1-v^{i} v_{i}}=1 / \sqrt{1-v^{2}}$ the Lorentz factor and the 4-vector $\boldsymbol{b}$ has components

$$
b^{\alpha} \equiv\left\{W(\vec{v} \cdot \vec{B}), \frac{\vec{B}}{W}+W(\vec{v} \cdot \vec{B}) \vec{v}\right\} .
$$

Here $\vec{B}$ is the magnetic field 3-vector and

$$
b^{2} \equiv b^{i} b_{i}=\frac{B^{2}}{W^{2}}+(\vec{v} \cdot \vec{B})^{2}=2 p_{\mathrm{m}}
$$

The general relativistic equations of MHD are then simply obtained after requiring the conservation of baryon number

$$
\nabla_{\mu}\left(\rho u^{\mu}\right)=0,
$$

where $\nabla$ represents a covariant derivative, the conservation of energy and momentum

$$
\nabla_{\mu} T^{\mu \nu}=0,
$$


together with the relevant pair of Maxwell equations. If the fluid is assumed to have an infinite electrical conductivity (i.e. ideal MHD limit), the Maxwell equations reduce to $\partial_{[\alpha} F_{\beta \gamma]}=0$, where $\boldsymbol{F}$ is the Faraday tensor and the square brackets refer to antisymmetrised indices. Using the definition (2.2), the Maxwell equations can be simply written as

$$
\nabla_{\mu}\left(b^{\mu} u^{\nu}-u^{\mu} b^{\nu}\right)=0 .
$$

The system of equations (2.4)-2.6 is completed with an equation of state (EOS) relating the pressure to the rest-mass density and/or to the energy density. Although hereafter we will use an ideal-gas EOS: $p_{\mathrm{g}}=\rho \epsilon(\Gamma-1)$, where $\Gamma$ is the polytropic index, the procedure described for the solution of the Riemann problem is valid for a generic EOS.

We next assume that the system has a planar-symmetry, i.e. that in a Cartesian coordinate system $(t, x, y, z)$ all the variables depend only on $t$ and $x$, and that the spacetime is flat so that covariant derivatives in equations (2.4)-(2.6) can be replaced by partial derivatives and $A^{i}=A_{i}$ for any 3 -vector $\vec{A}$. In this case, the complete set of MHD equations can be written as a set of first-order partial differential equations in a flux-conservative form

$$
\frac{\partial \mathbf{U}}{\partial t}+\frac{\partial \mathbf{F}}{\partial x}=0
$$

where $\mathbf{U}$ and $\mathbf{F}$ are respectively the vectors of conserved quantities and fluxes, defined as

$$
\mathbf{U} \equiv\left(\begin{array}{c}
D \\
\tau-b^{0} b^{0} \\
S^{x}-b^{0} b^{x} \\
S^{y}-b^{0} b^{y} \\
S^{z}-b^{0} b^{z} \\
B^{y} \\
B^{z}
\end{array}\right), \quad \mathbf{F} \equiv\left(\begin{array}{c}
D v^{x} \\
S^{x}-b^{0} b^{x}-D v^{x} \\
S^{x} v^{x}+p-b^{x} b^{x} \\
S^{y} v^{x}-b^{x} b^{y} \\
S^{z} v^{x}-b^{x} b^{z} \\
B^{y} v^{x}-B^{x} v^{y} \\
B^{z} v^{x}-B^{x} v^{z}
\end{array}\right)
$$

and where the following definitions have been used

$$
\begin{aligned}
\tau & \equiv w W^{2}-p-D, \\
D & \equiv \rho W, \\
S^{j} & \equiv \rho h W^{2} v^{j}, \\
p & \equiv p_{\mathrm{g}}+p_{\mathrm{m}}=p_{\mathrm{g}}+\frac{1}{2} b^{2}, \\
w & \equiv \rho h, \\
h & \equiv h_{\mathrm{g}}+\frac{b^{2}}{\rho}=1+\epsilon+\frac{p_{\mathrm{g}}}{\rho}+\frac{b^{2}}{\rho},
\end{aligned}
$$

where $h$ is the total specific enthalpy and $h_{\mathrm{g}}$ the one of the gas only.

Note that the divergence-free condition for the magnetic field and the Maxwell equation for the evolution of the $x$-component of the magnetic field imply that $\partial_{t} B^{x}=0=\partial_{x} B^{x}$, i.e. $B^{x}$ is uniform in space, constant in time and thus always maintains its initial values.

\section{Strategy of Solution}

The general Riemann problem in relativistic MHD consists of a set of seven nonlinear waves: two fast-waves (FW), two slow-waves ( $\mathrm{SW}$ ), two Alfvèn-waves (AW), and a contact discontinuity (CD) at which only the density may be discontinuous. The fast and slow nonlinear waves can be either shocks or rarefaction waves, depending on the change in the pressure and in the norm of the magnetic field across the wave. 


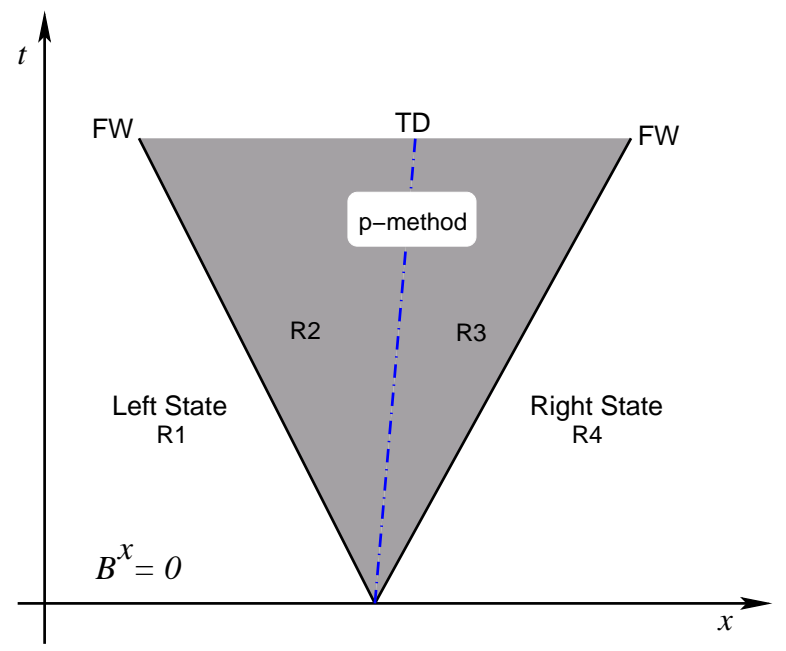

FIGURE 1. Spacetime structure of the MHD Riemann problem in the case in which the magnetic field has tangential components only, i.e. $B^{x}=0$. The "Riemann-fan" in this case is composed of only two fast-waves (FW) and of a central tangential discontinuity (TD), thus resembling structure of the Riemann problem in pure hydrodynamics. Indicated with R1-R4 are the 4 different regions into which the Riemann problem can be decomposed, each representing a different state.

Building on the experience with relativistic hydrodynamics, our general strategy in the search for the solution consists of expressing all of the variables after each wave as functions of the values of the same variables ahead of the wave and of an unknown variable behind the wave. When considering the Riemann problem in relativistic hydrodynamics, in fact, the solution is found after expressing all of the quantities behind the wave as functions of the value of the pressure at the contact discontinuity. In this way, the problem is reduced to the search for the value of the pressure that satisfies the jump conditions at the contact discontinuity.

When considering the Riemann problem in relativistic MHD, on the other hand, two different cases need to be distinguished. Assuming the initial discontinuity to have normal along the $x$-axis, the initial magnetic field in this direction can either be zero (i.e. $B^{x}=0$ ) or not (i.e. $B^{x} \neq 0$ ). In the first case, the structure of the solution is very similar to the hydrodynamical one, with only two fast waves and a tangential discontinuity along which only the total pressure and the $x$ component of the velocity are continuous. The spacetime structure of the Riemann problem in this case is sketched in Figure1 where the "Riemann-fan" is shown to be composed of only two fast-waves (FW) and of a central tangential discontinuity (TD). Because of this analogy, the numerical solution of the Riemann problem when $B^{x}=0$ follows the same procedure implemented in relativistic hydrodynamics. We refer to this as the "total-pressure approach" or simply, the "p-method".

A detailed investigation of the exact solution of the Riemann problem with tangential magnetic fields and when the additional condition $\vec{v} \cdot \vec{B}=0$ is imposed, has been recently proposed by Romero et al. (2005). Among the many points discussed, this work has shown that when $B^{x}=0=\vec{v} \cdot \vec{B}$ the Riemann problem in relativistic MHD can be assimilated to the one in relativistic hydrodynamics and that all of the corrections introduced by the magnetic field can be incorporated in the definition of a new, effective EOS.

In the second case, on the other hand, the Riemann problem is considerably more complex and all of the seven waves are allowed to form when the initial discontinuity is removed. The spacetime structure of the Riemann problem in this case is sketched in Figure 2 where the 


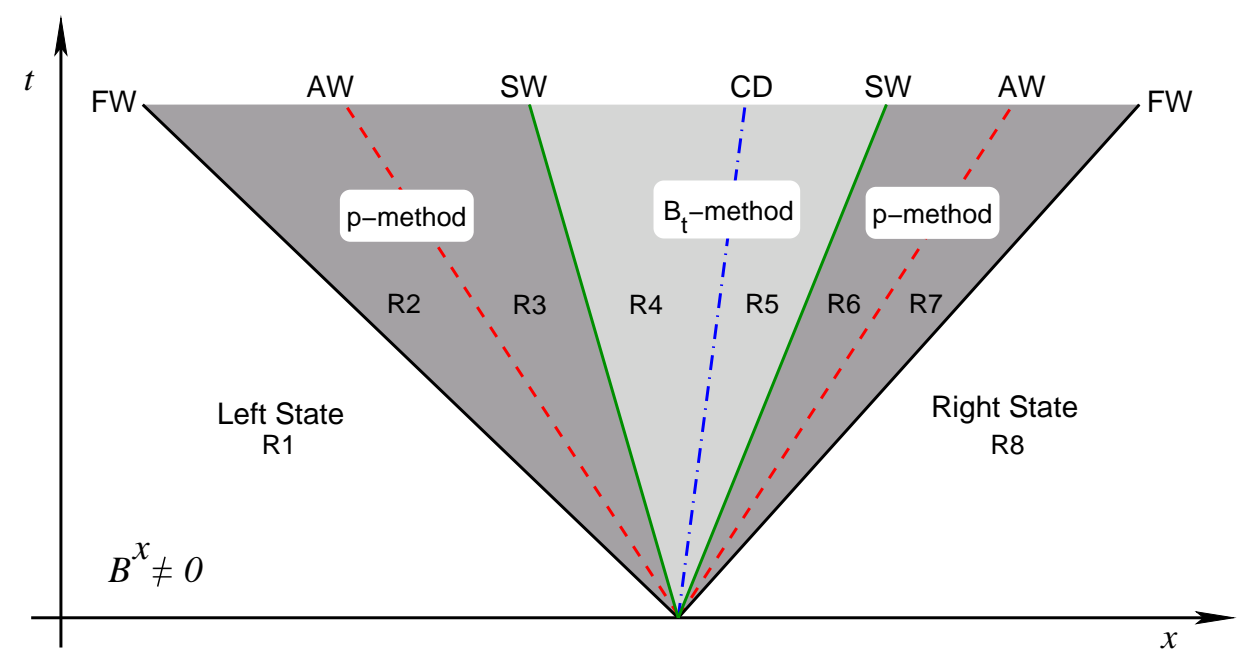

FIGURE 2. Spacetime structure of the MHD Riemann problem in the general case in which the magnetic field has also a normal component, i.e. $B^{x} \neq 0$. The "Riemann-fan" is here composed of two fast-waves (FW), of two Alfvèn waves (AW), of two slow-waves (SW) and of a central contact discontinuity (CD). Indicated with R1-R8 are the 8 different regions into which the Riemann problem can be decomposed, each representing a different state. Indicated are also the different methods used to compute the solutions in the different regions (i.e. $B_{t}$-method in regions R4 and R5 and $p$-method in regions R2-R3 and R6-R7).

"Riemann-fan" is shown to be composed of two fast-waves (FW), of two Alfvèn waves (AW), of two slow-waves (SW) and of a central contact discontinuity (CD).

It is important to bear in mind that across the Alfvèn discontinuities only the total pressure, the gas pressure and the density are continuous, while there could be jumps in the other quantities. As a result, if the total pressure is used as unknown, there would be three different values for the total pressure (two between the fast and the slow-waves and one between the two slow-waves) but five conditions to be satisfied at the contact discontinuity: the continuity of the three components of the velocity and the continuity of the tangential components of the magnetic field. The resulting system of five equations in three unknowns is over-constrained and there is no guarantee that a global convergent solution is found at the contact discontinuity. Indeed, experience has shown that small numerical imprecisions at the level of round-off errors are in general sufficient to prevent the simultaneous solution of the five constraints.

To circumvent this difficulty and inspired by the procedure followed in the exact solution of the corresponding Riemann solver in nonrelativistic MHD (Ryu \& Jones 1995), when $B^{x} \neq 0$ we have implemented a "hybrid" approach in which the total pressure is used as the unknown variable between the fast and the slow waves (i.e. in regions R2-R3, and R6-R7 of Figure 2), while the tangential components of the magnetic fields $\left(B^{y}\right.$ and $\left.B^{z}\right)$ is used between the slow waves (i.e. in regions R4-R5 of Figure 2). In this way, the continuity of the tangential components of the magnetic field $B^{y}$ and $B^{z}$ is automatically guaranteed through the contact discontinuity and only the continuity of the total pressure and of the three components of the velocity needs to be satisfied. The resulting system consists of four equations in four unknowns and, being closed, it can be solved numerically through root-finding techniques for nonlinear system of equations (e.g. using a Newton-Raphson method). We refer to this as the "tangential magnetic field approach" or simply, the " $B_{t}$-method".

As mentioned in the Introduction, hereafter we will assume that the Riemann problem has a solution and that this is unique. As a result, we will not discuss in any detail compound waves which seem to develop in the numerical solution of some special initial states (one of these is 
shown in Section 6.2.1 and whose admissibility as solution of the Riemann problem is still debated.

\section{Total-Pressure Approach: " $p$-method"}

In the following Sections we describe in detail the approach in which we calculate all of the variables in the Riemann fan using as unknown the total pressure, i.e. the $p$-method. Different set of equations will be derived according to whether the solution is across a shock or a rarefaction wave.

\subsection{Solution across a shock front}

Consider $\Sigma$ to be a hypersurface in flat spacetime across which $\rho, \boldsymbol{u}$ and $\boldsymbol{T}$ are discontinuous. Let also $\boldsymbol{n}$ be the unit 4-vector normal to $\Sigma$ so that the Rankine-Hugoniot conditions for relativistic MHD can be expressed as

$$
\begin{aligned}
{\left[\left[\rho u^{\alpha}\right]\right] n_{\alpha} } & =0, \\
{\left[\left[T^{\alpha \beta}\right]\right] n_{\alpha} } & =0, \\
{\left[\left[b^{\alpha} u^{\beta}-u^{\alpha} b^{\beta}\right]\right] n_{\alpha} } & =0,
\end{aligned}
$$

where we use the double-bracket notation to express the jump of a quantity $F$ across the hypersurface $\Sigma$, i.e.

$$
[[F]] \equiv F_{a}-F_{b}
$$

where $F_{a}$ and $F_{b}$ are respectively the values ahead $(a)$ and behind $(b)$ the shock.

In particular, if $\Sigma$ is the 4-dimensional hypersurface describing the evolution of a shock wave normal to the $x$-axis, the unitary condition on $\boldsymbol{n}$ can be used to derive the components

$$
n^{\alpha}=W_{s}\left(V_{s}, 1,0,0\right),
$$

where $V_{s}$ is the coordinate velocity of the shock, $W_{s} \equiv\left(1-V_{s}^{2}\right)^{-1 / 2}$ its Lorentz factor, and we can rewrite equations 4.1 4.3. explicitly as

$$
\begin{aligned}
{[[J]] \equiv\left[\left[\rho W\left(V_{s}-v^{x}\right) W_{s}\right]\right] } & =0, \\
{\left[\left[b^{0} b^{0}-\tau\right]\right] V_{s}+\left[\left[S^{x}-b^{0} b^{x}-D v^{x}\right]\right] } & =0, \\
{\left[\left[b^{0} b^{x}-S^{x}\right]\right] V_{s}+\left[\left[S^{x} v^{x}+p-b^{x} b^{x}\right]\right] } & =0, \\
{\left[\left[b^{0} b^{y}-S^{y}\right]\right] V_{s}+\left[\left[S^{y} v^{x}-b^{x} b^{y}\right]\right] } & =0, \\
{\left[\left[b^{0} b^{z}-S^{z}\right]\right] V_{s}+\left[\left[S^{z} v^{x}-b^{x} b^{z}\right]\right] } & =0, \\
{\left[\left[B^{x}\right]\right] } & =0, \\
{\left[\left[B^{y}\right]\right] V_{s}+\left[\left[B^{x} v^{y}-v^{x} B^{y}\right]\right] } & =0, \\
{\left[\left[B^{z}\right]\right] V_{s}+\left[\left[B^{x} v^{z}-v^{x} B^{z}\right]\right] } & =0,
\end{aligned}
$$

where $J$ is the (rest) mass flux across the shock.

After a number of tedious but otherwise straightforward algebraic manipulations, equations 
4.5 -4.12 can be recast as

$$
\begin{aligned}
{\left[\left[v^{x}\right]\right]+\frac{J}{W_{s}}\left[\left[\frac{1}{D}\right]\right] } & =0, \\
\frac{J}{W_{s}}\left[\left[\frac{W^{2} \eta^{2}}{D}\right]\right]-B^{x}[[\eta]]-\frac{J}{W_{s}}\left[\left[\frac{\tau}{D}\right]\right]+\left[\left[p v^{x}\right]\right] & =0, \\
\frac{J B^{x}}{W_{s}}\left[\left[\frac{\eta}{D}\right]\right]-B^{x}\left[\eta v^{x}\right]+\frac{J}{W_{s}}\left[\left[\frac{W^{2} \eta^{2} v^{x}}{D}\right]\right]-\left[\left[\frac{B_{x}^{2}}{W^{2}}\right]\right]-\frac{J}{W_{s}}\left[\left[\frac{S^{x}}{D}\right]\right]+[[p]] & =0, \\
\frac{J}{W_{s}}\left[\left[\frac{\eta B^{y}}{D}\right]\right]+\frac{J}{W_{s}}\left[\left[\frac{W^{2} \eta^{2} v^{y}}{D}\right]\right]-B^{x}\left[\left[\frac{B^{y}}{W^{2}}\right]\right]-B^{x}\left[\left[\eta v^{y}\right]\right]-\frac{J}{W_{s}}\left[\left[\frac{S^{y}}{D}\right]\right] & =0, \\
\frac{J}{W_{s}}\left[\left[\frac{\eta B^{z}}{D}\right]\right]+\frac{J}{W_{s}}\left[\left[\frac{W^{2} \eta^{2} v^{z}}{D}\right]\right]-B^{x}\left[\left[\frac{B^{z}}{W^{2}}\right]\right]-B^{x}\left[\left[\eta v^{z}\right]\right]-\frac{J}{W_{s}}\left[\left[\frac{S^{z}}{D}\right]\right] & =0,\left[\left[B^{x}\right]\right]=0, \\
\frac{J}{W_{s}}\left[\left[\frac{B^{y}}{D}\right]\right]+B^{x}\left[\left[v^{y}\right]\right] & =0, \\
\frac{J}{W_{s}}\left[\left[\frac{B^{z}}{D}\right]\right]+B^{x}\left[\left[v^{z}\right]\right] & =0,
\end{aligned}
$$

where we have defined $\eta \equiv \vec{v} \cdot \vec{B}$ and exploited the property

$$
\left[\left[F\left(V_{s}-v^{x}\right)\right]\right]=\frac{J}{W_{s}}\left[\left[\frac{F}{D}\right]\right],
$$

valid for any scalar quantity $F$.

The next step to take is to express all of the variables as functions of $J$ and $p_{b}$ only. We start by using equation 4.13 to obtain

$$
\frac{1}{D_{b}}=\left(v_{a}^{x}-v_{b}^{x}\right) \frac{W_{s}}{J}+\frac{1}{D_{a}}
$$

so that equation 4.14 yields

$$
\frac{\tau_{b}}{D_{b}}=-\frac{W_{a}^{2} \eta_{a}^{2}}{D_{a}}+\frac{W_{b}^{2} \eta_{b}^{2}}{D_{b}}+\frac{W_{s}}{J}\left[B^{x}\left(\eta_{a}-\eta_{b}\right)-p_{a} v_{a}^{x}+p_{b} v_{b}^{x}\right]+\frac{\tau_{a}}{D_{a}},
$$

which depends on $v_{b}^{x}, p_{b}$ but also on $B_{b}^{y}, B_{b}^{z}, v_{b}^{y}, v_{b}^{z}$. To remove the dependence from these latter quantities we employ equations (4.19) and (4.20) to obtain $B_{b}^{y}$ and $B_{b}^{z}$ as functions of $v_{b}^{x}, v_{b}^{y} v_{b}^{z}$ and $p_{b}$, i.e.

$$
\begin{aligned}
& B_{b}^{y}=D_{b}\left(\frac{B_{a}^{y}}{D_{a}}+\frac{W_{s}}{J} B^{x} v_{a}^{y}-\frac{W_{s}}{J} B^{x} v_{b}^{y}\right), \\
& B_{b}^{z}=D_{b}\left(\frac{B_{a}^{z}}{D_{a}}+\frac{W_{s}}{J} B^{x} v_{a}^{z}-\frac{W_{s}}{J} B^{x} v_{b}^{z}\right) .
\end{aligned}
$$

We can now solve equation (4.15) and finally obtain $v_{b}^{x}$ as a function of $v_{b}^{y}, v_{b}^{z}, p_{b}$ and $J$

$$
\begin{gathered}
v_{b}^{x}=\frac{D_{a}\left\{B_{x}^{2} W_{s}+W_{a}^{2}\left[W_{s}\left(p_{b}-p_{a}\right)-B_{x}^{2} W_{s}\left(1-v_{a}^{y} v_{b}^{y}-v_{a}^{z} v_{b}^{z}\right)+v_{a}^{x}\left(J+B^{x} W_{s} \eta_{a}\right)\right]\right\}}{W_{a}^{2}\left\{D_{a}\left[J-W_{s}\left(B_{x}^{2}+p_{a}-p_{b}\right) v_{a}^{x}+B^{x} W_{s} \eta_{a}\right]-J\left(B_{x}^{2}-p_{b}+W_{a}^{2} \eta_{a}^{2}-\tau_{a}\right)\right\}}+ \\
\frac{J\left[B^{x}\left(B_{a}^{y} v_{b}^{y}+B_{a}^{z} v_{b}^{z}-\eta_{a}\right)+v_{a}^{x}\left(p_{a}-W_{a}^{2} \eta_{a}^{2}+\tau_{a}\right)\right]}{D_{a}\left[J-W_{s}\left(B_{x}^{2}+p_{a}-p_{b}\right) v_{a}^{x}+B^{x} W_{s} \eta_{a}\right]-J\left(B_{x}^{2}-p_{b}+W_{a}^{2} \eta_{a}^{2}-\tau_{a}\right)},
\end{gathered}
$$


where it should be noted that equation (4.25) reduces to the corresponding hydrodynamical expression in the limit of $\vec{B}=0$ [cf. equation (4.12) of Pons et al. (2000) or equation (3.13) of Rezzolla et al. (2003)]. Note also that using equations (4.16 and 4.17) it is possible to obtain expressions for $v_{b}^{y}$ and $v_{b}^{z}$ in terms of the post-shock quantities $p$ and $J$; the corresponding expressions are rather lengthy and uninspiring; for this reason we report them in Appendix $\mathrm{A}$

When all of the post-shock quantities are expressed as functions of only $p_{b}$ and $J$ (i.e. $V_{s}$ ), it is still necessary to express $V_{s}$ as function of the post-shock pressure $p_{b}$. To do this we follow Pons et al. (2000) and use the original jump conditions (4.1), 4.2) and (4.3) to obtain

$$
\begin{aligned}
& {[[p]]+J^{2}\left[\left[\frac{h_{\mathrm{g}}}{\rho}\right]\right]=0,} \\
& {\left[\left[h_{\mathrm{g}}^{2}\right]\right]-\left(\left(\frac{h_{\mathrm{g}}}{\rho}\right)\right)[[p]]-H\left[\left[b^{2}\right]\right]+2\left[\left[b^{2} \frac{h_{\mathrm{g}}}{\rho}\right]\right]-2 J^{2} H\left[\left[\frac{h_{\mathrm{g}}}{\rho}\right]\right]=0,}
\end{aligned}
$$

where $((F)) \equiv F_{a}+F_{b}$ and $H \equiv B_{n}^{2} / J^{2}-b^{2} / \rho^{2}$ is a shock invariant quantity (i.e. $[[H]]=0$, Anile 1989). Note that $B_{n}$ is not just the normal component of the magnetic field but, rather, the projection of $\boldsymbol{b}$ along $\boldsymbol{n}$, i.e.

$$
B_{n} \equiv b^{\mu} n_{\mu}=-\frac{\eta}{\rho} J+\frac{W_{s}}{W} B^{x}
$$

Equation (4.27) is also known as the Lichnerowicz adiabat, and represents the relativistic MHD counterpart of the Hugoniot adiabat.

A couple of remarks should be made. Firstly, equations 4.26-4.27 can be used for fast and slow shocks but not for an Alfvèn discontinuity. In this case, in fact, $[[h / \rho]]=0$, equations (4.26-4 (4.27) are simple identities and the shock velocity $V_{s}$ is trivially given by the local Alfvèn velocity $V_{A}$. Secondly, for purely hydrodynamical shocks it is possible to find an analytic expression for $V_{s}$ as a function of the post-shock pressure [cf. equation (4.14) of Pons et al. (2000)]. In relativistic MHD, however, the corresponding analytic expression has not been found and equation (4.26) needs to be solved numerically using a standard root-finding algorithm, but also increasing the computational costs considerably. To guarantee that we are using the right shock velocity, the root is searched in the approriate physical interval, i.e. $\left|V_{s}\right| \in\left(\left|V_{A}\right|, 1\right)$ for fast shocks and $\left|V_{s}\right| \in\left(\left|v^{x}\right|,\left|V_{A}\right|\right)$ for slow shocks.

\subsection{Solution across a rarefaction wave}

Rarefaction waves are self-similar solutions of the flow equations, i.e. equations in which all of the fluid quantities depend on $x$ and $t$ through the combination $\xi \equiv x / t$. Using this as the independent variable, the set of partial differential MHD equations can be rewritten as the following 
set of ordinary differential equations (ODEs)

$$
\begin{aligned}
\xi \frac{\mathrm{d} D}{\mathrm{~d} \xi}-\frac{\mathrm{d}\left(D v^{x}\right)}{\mathrm{d} \xi} & =0 \\
\xi \frac{\mathrm{d}\left(\tau-b^{0} b^{0}\right)}{\mathrm{d} \xi}-\frac{\mathrm{d}\left(S^{x}-b^{0} b^{x}-D v^{x}\right)}{\mathrm{d} \xi} & =0 \\
\xi \frac{\mathrm{d}\left(S^{x}-b^{0} b^{x}\right)}{\mathrm{d} \xi}-\frac{\mathrm{d}\left(S^{x} v^{x}+p-b^{x} b^{x}\right)}{\mathrm{d} \xi} & =0 \\
\xi \frac{\mathrm{d}\left(S^{y}-b^{0} b^{y}\right)}{\mathrm{d} \xi}-\frac{\mathrm{d}\left(S^{y} v^{x}-b^{x} b^{y}\right)}{\mathrm{d} \xi} & =0 \\
\xi \frac{\mathrm{d}\left(S^{z}-b^{0} b^{z}\right)}{\mathrm{d} \xi}-\frac{\mathrm{d}\left(S^{z} v^{x}-b^{x} b^{z}\right)}{\mathrm{d} \xi} & =0, \\
\xi \frac{\mathrm{d} B^{x}}{\mathrm{~d} \xi} & =0 \\
\xi \frac{\mathrm{d} B^{y}}{\mathrm{~d} \xi}-\frac{\mathrm{d}\left(B^{y} v^{x}-B^{x} v^{y}\right)}{\mathrm{d} \xi} & =0 \\
\xi \frac{\mathrm{d} B^{z}}{\mathrm{~d} \xi}-\frac{\mathrm{d}\left(B^{z} v^{x}-B^{x} v^{z}\right)}{\mathrm{d} \xi} & =0 .
\end{aligned}
$$

Equation 4.29] can be further decomposed as

$$
\left(v^{x}-\xi\right) \frac{\mathrm{d} \rho}{\mathrm{d} \xi}+\rho\left[\left(v^{x}-\xi\right) W^{2} v^{x}+1\right] \frac{\mathrm{d} v^{x}}{\mathrm{~d} \xi}+\left(v^{x}-\xi\right) \rho W^{2} v^{y} \frac{\mathrm{d} v^{y}}{\mathrm{~d} \xi}+\left(v^{x}-\xi\right) \rho W^{2} v^{z} \frac{\mathrm{d} v^{z}}{\mathrm{~d} \xi}=0,
$$

while combining equation (4.30) with equations 4.31-4.33) provides us with the relations

$$
\begin{aligned}
& w W^{2}\left(v^{x}-\xi\right) \frac{\mathrm{d} v^{x}}{\mathrm{~d} \xi}+\left(1-\xi v^{x}\right) \frac{\mathrm{d} p}{\mathrm{~d} \xi}-v^{x} \xi \frac{\mathrm{d}\left(b^{0} b^{0}\right)}{\mathrm{d} \xi}+\left(v^{x}+\xi\right) \frac{\mathrm{d}\left(b^{0} b^{x}\right)}{\mathrm{d} \xi}-\frac{\mathrm{d}\left(b^{x} b^{x}\right)}{\mathrm{d} \xi}=0 \\
& w W^{2}\left(v^{x}-\xi\right) \frac{\mathrm{d} v^{y}}{\mathrm{~d} \xi}-\xi v^{y} \frac{\mathrm{d} p}{\mathrm{~d} \xi}-\xi v^{y} \frac{\mathrm{d}\left(b^{0} b^{0}\right)}{\mathrm{d} \xi}+v^{y} \frac{\mathrm{d}\left(b^{0} b^{x}\right)}{\mathrm{d} \xi}+\xi \frac{\mathrm{d}\left(b^{0} b^{y}\right)}{\mathrm{d} \xi}-\frac{\mathrm{d}\left(b^{x} b^{y}\right)}{\mathrm{d} \xi}=0 \\
& w W^{2}\left(v^{x}-\xi\right) \frac{\mathrm{d} v^{z}}{\mathrm{~d} \xi}-\xi v^{z} \frac{\mathrm{d} p}{\mathrm{~d} \xi}-\xi v^{z} \frac{\mathrm{d}\left(b^{0} b^{0}\right)}{\mathrm{d} \xi}+v^{z} \frac{\mathrm{d}\left(b^{0} b^{x}\right)}{\mathrm{d} \xi}+\xi \frac{\mathrm{d}\left(b^{0} b^{z}\right)}{\mathrm{d} \xi}-\frac{\mathrm{d}\left(b^{x} b^{z}\right)}{\mathrm{d} \xi}=0 .
\end{aligned}
$$

Finally, rewriting the definition of the local sound speed

$$
\left.c_{s}^{2} \equiv \frac{1}{h_{g}} \frac{\partial p_{\mathrm{g}}}{\partial \rho}\right|_{s}
$$

where $s$ is the specific entropy, in terms of the self-similar variable

$$
\frac{\mathrm{d} p_{\mathrm{g}}}{\mathrm{d} \xi}=h_{g} c_{s}^{2} \frac{\mathrm{d} \rho}{\mathrm{d} \xi}
$$

and collecting the different terms in equations (4.29)-4.36, we obtain the following system of seven ODEs in the seven variables $\rho, p, v^{x}, v^{y}, v^{z}, B^{y}, B^{z}$, fully determining the solution across 
a rarefaction wave

$$
\begin{aligned}
& 0=\left(v^{x}-\xi\right) \frac{\mathrm{d} \rho}{\mathrm{d} \xi}+\rho\left[\left(v^{x}-\xi\right) W^{2} v^{x}+1\right] \frac{\mathrm{d} v^{x}}{\mathrm{~d} \xi}+\left(v^{x}-\xi\right) \rho W^{2} v^{y} \frac{\mathrm{d} v^{y}}{\mathrm{~d} \xi}+\left(v^{x}-\xi\right) \rho W^{2} v^{z} \frac{\mathrm{d} v^{z}}{\mathrm{~d} \xi}, \\
& 0=\frac{\mathrm{d} p}{\mathrm{~d} \xi}-h_{g} c_{s}^{2} \frac{\mathrm{d} \rho}{\mathrm{d} \xi}+\left(B^{2} v^{x}-B^{x} \eta\right) \frac{\mathrm{d} v^{x}}{\mathrm{~d} \xi}+\left(B^{2} v^{y}-B^{y} \eta\right) \frac{\mathrm{d} v^{y}}{\mathrm{~d} \xi}+\left(B^{2} v^{z}-B^{z} \eta\right) \frac{\mathrm{d} v^{z}}{\mathrm{~d} \xi}- \\
& \left(\frac{B^{y}}{W^{2}}+v^{y} \eta\right) \frac{\mathrm{d} B^{y}}{\mathrm{~d} \xi}-\left(\frac{B^{z}}{W^{2}}+v^{z} \eta\right) \frac{\mathrm{d} B^{z}}{\mathrm{~d} \xi}, \\
& 0=\left(1-v^{x} \xi\right) \frac{\mathrm{d} p}{\mathrm{~d} \xi}+\left[B_{x}^{2}\left(v^{x}+\xi\right)-2 B^{x} \eta+W^{2}\left(v^{x}-\xi\right)\left(w-\eta^{2}\right)\right] \frac{\mathrm{d} v^{x}}{\mathrm{~d} \xi}+ \\
& 2 B_{x}^{2}\left\{\left[v^{y}+\frac{B^{y}\left(\xi-v^{x}\right)}{2 B^{x}}\right] \frac{\mathrm{d} v^{y}}{\mathrm{~d} \xi}+\left[v^{z}+\frac{B^{z}\left(\xi-v^{x}\right)}{2 B^{x}}\right] \frac{\mathrm{d} v^{z}}{\mathrm{~d} \xi}-\frac{\left(v^{x}-\xi\right)}{2 B^{x}}\left[v^{y} \frac{\mathrm{d} B^{y}}{\mathrm{~d} \xi}+v^{z} \frac{\mathrm{d} B^{z}}{\mathrm{~d} \xi}\right]\right\}, \\
& 0=\xi v^{y} \frac{\mathrm{d} p}{\mathrm{~d} \xi}- \\
& B^{x} B^{y}\left\{\left[\left(v^{x}+\xi\right)-\frac{\eta}{B^{x}}\right] \frac{\mathrm{d} v^{x}}{\mathrm{~d} \xi}+\left[2 v^{y}+\frac{B^{y}\left(\xi-v^{x}\right)}{B^{x}}+\frac{W^{2}\left(v^{x}-\xi\right)\left(w-\eta^{2}\right)}{B^{x} B^{y}}-\frac{\eta}{B^{y}}\right] \frac{\mathrm{d} v^{y}}{\mathrm{~d} \xi}\right\}- \\
& B^{y}\left[2 B^{x} v^{z}+B^{z}\left(\xi-v^{x}\right)\right] \frac{\mathrm{d} v^{z}}{\mathrm{~d} \xi}+\left[\frac{B^{x}+W^{2}\left(v^{x}-\xi\right)\left(B^{y} v^{y}+\eta\right)}{W^{2}}\right] \frac{\mathrm{d} B^{y}}{\mathrm{~d} \xi}+B^{y} v^{z}\left(v^{x}-\xi\right) \frac{\mathrm{d} B^{z}}{\mathrm{~d} \xi}, \\
& 0=\xi v^{z} \frac{\mathrm{d} p}{\mathrm{~d} \xi}- \\
& B^{x} B^{z}\left\{\left[\left(v^{x}+\xi\right)-\frac{\eta}{B^{x}}\right] \frac{\mathrm{d} v^{x}}{\mathrm{~d} \xi}+\left[2 v^{z}+\frac{B^{z}\left(\xi-v^{x}\right)}{B^{x}}+\frac{W^{2}\left(v^{x}-\xi\right)\left(w-\eta^{2}\right)}{B^{x} B^{z}}-\frac{\eta}{B^{z}}\right] \frac{\mathrm{d} v^{z}}{\mathrm{~d} \xi}\right\}- \\
& B^{z}\left[2 B^{x} v^{y}+B^{y}\left(\xi-v^{x}\right)\right] \frac{\mathrm{d} v^{y}}{\mathrm{~d} \xi}+B^{z} v^{y}\left(v^{x}-\xi\right) \frac{\mathrm{d} B^{y}}{\mathrm{~d} \xi}+\left[\frac{B^{x}+W^{2}\left(v^{x}-\xi\right)\left(B^{z} v^{z}+\eta\right)}{W^{2}}\right] \frac{\mathrm{d} B^{z}}{\mathrm{~d} \xi}, \\
& 0=B^{y} \frac{\mathrm{d} v^{x}}{\mathrm{~d} \xi}-B^{x} \frac{\mathrm{d} v^{y}}{\mathrm{~d} \xi}+\left(v^{x}-\xi\right) \frac{\mathrm{d} B^{y}}{\mathrm{~d} \xi} \\
& 0=B^{z} \frac{\mathrm{d} v^{x}}{\mathrm{~d} \xi}-B^{x} \frac{\mathrm{d} v^{z}}{\mathrm{~d} \xi}+\left(v^{x}-\xi\right) \frac{\mathrm{d} B^{z}}{\mathrm{~d} \xi} .
\end{aligned}
$$

The system of equations 4.43 - 4.49 can be recast into a simple matrix form and non-trivial similarity solutions exist only if the determinant of the matrix of coefficients is zero. This condition leads to a quartic equation in the self-similar variable $\xi$

$$
\begin{aligned}
& \tilde{b}_{x}^{2} c_{s}^{2}-\zeta^{2} v_{x}^{2} W^{2}-\left(\zeta^{2}-1\right) v_{x}^{4} W^{4}+\left[2 \zeta^{2} v_{x} W^{2}-2 \tilde{b}^{0} \tilde{b}_{x} c_{s}^{2}+4\left(\zeta^{2}-1\right) v_{x}^{3} W^{4}\right] \xi+ \\
& {\left[\left(\tilde{b}^{0}-\tilde{b}_{x}\right)\left(\tilde{b}^{0}+\tilde{b}_{x}\right) c_{s}^{2}+\zeta^{2}\left(v_{x}^{2}-1\right) W^{2}-6\left(\zeta^{2}-1\right) v_{x}^{2} W^{4}\right] \xi^{2}+} \\
& {\left[2 \tilde{b}^{0} \tilde{b}_{x} c_{s}^{2}-2 \zeta^{2} v_{x} W^{2}+4\left(\zeta^{2}-1\right) v_{x} W^{4}\right] \xi^{3}+\left[W^{4}+W^{2} \zeta^{2}\left(1-W^{2}\right)-\left(\tilde{b}^{0}\right)^{2} c_{s}^{2}\right] \xi^{4}=0, }
\end{aligned}
$$

where

$$
\tilde{b} \equiv \frac{b}{\sqrt{w}}, \quad \text { and } \quad \zeta^{2} \equiv c_{s}^{2}+\tilde{b}^{2}\left(1-c_{s}^{2}\right)
$$


and whose roots coincide with the eigenvalues of the original system of equations (2.4)- 2.6. When $B^{x}=0$, equation (4.50) reduces to a second-order equation whose roots provide the velocities of the left and right-going fast-waves. In the more general case when $B^{x} \neq 0$, however, the quartic cannot be recast as the product of two quadratic equations (as it is the case in Newtonian hydrodynamics) and the solution must be found numerically. The corresponding roots provide the velocities of the left and right-going slow and fast magnetosonic rarefaction waves, respectively.

Using the appropriate root for $\xi$, the system of ODEs (4.43)-4.49) can be rewritten in terms of the total pressure to obtain a new system of six ODEs to be integrated from the value of pressure ahead the rarefaction to the one behind it $\dagger$. The explicit expressions of these equations are rather lengthy and do not provide any important information; for this reason we report them in Appendix B

\subsection{Solution across an Alfvèn discontinuity}

The solution across Alfvèn discontinuities is found by imposing the continuity of $\rho$ and $p$ and then solving the system of equations (4.15)-(4.17) and (4.19)-(4.20), using $V_{s}=V_{A}$, where $V_{A} \equiv$ $v_{x}+B_{x} /\left[W^{2}(\eta \mp \sqrt{w})\right]$ is the Alfvèn velocity for left $(-)$ and right $(+)$ going waves, respectively. Since $\rho$ and $p$ are continuous across the Alfvèn discontinuity, a solution needs to be found only for the three components of $\vec{v}$ and for the tangential components of the magnetic field $B_{y}$ and $B_{z}$. In general, and because no analytic solution was found, we solve the corresponding system of equations (4.15)-(4.17), (4.19)- 4.20) numerically with a Newton-Raphson scheme. No major difficulties have been found in determining an accurate solution provided that the waves are all well separated and that a sufficiently accurate initial guess is provided ( $c f$. solution in Figure 12). For the latter we have used an approximate Riemann solver based on the Harten-Lax-van LeerEinfeldt (HLLE) algorithm (Harten et al. 1983, Einfeldt 1988) and a moderate truncation error (i.e. using about 800 gridpoints for the tests reported here). However, considerable difficulties have been encountered if the waves are very close to each other. This is the case, for instance, of test number 5 of Balsara (2001), in which the left-going Alfvèn discontinuity and the leftgoing slow rarefaction wave have very similar propagation velocities ( $c f$. solution in Figure 11). The exact solution found in this case has a truncation error which is small, but larger that those reached in the other tests ( $c f$. data in Table 11].

\section{Tangential Magnetic Field Approach: " $\boldsymbol{B}_{t}$-method"}

As done in Sect. 4 in what follows we describe in detail the approach referred to as the $B_{t^{-}}$ method, in which we calculate all of the variables in the Riemann fan using as unknowns the values of the tangential components of the magnetic field, i.e. $B^{y}$ and $B^{z}$. As mentioned in the Introduction, much of the inspiration in the development and use of this method comes from the corresponding approach developed by Ryu and Jones (1995) in nonrelativistic MHD. However, important differences are also present.

In particular, in Newtonian MHD the problem can be solved using the norm of the tangential component of the magnetic field $B_{t} \equiv \sqrt{B_{y}^{2}+B_{z}^{2}}$ and the rotation angle $\psi \equiv \arctan \left(B^{z} / B^{y}\right)$ across Alfvèn discontinuities. This is because $B_{t}$ is conserved across Alfvèn discontinuities and $\psi$ is constant across fast and slow-waves (see Jeffrey 1966). As a result, the relevant system of equations is solved using as unknowns the values of $B_{t}$ in regions R2-R3, R4-R5, R6-R7 of the Riemann fan in Figure 2 and the angle $\psi$ in regions R3-R6. At the contact discontinuity it is then necessary to solve a system of four equations, given by the continuity of $\vec{v}$ and of $p$, in the same

$\dagger$ The number of equations to be solved reduces from seven to six because when using the total pressure as the self-similar variable one equation becomes then trivial, i.e. $d p / d p=1$. 
four unknowns. This can be solved using root-finding techniques such as the Newton-Raphson method. Finally, when $B_{x}=0$, the presence of only two fast waves and a tangential discontinuity makes the solution of the problem even simpler (see Ryu and Jones 1995 for details).

In relativistic MHD, on the other hand, the value of $B_{t}$ can be discontinuous across Alfvèn waves and the angle $\psi$ can vary across fast and slow-waves; it is then not possible to solve the system using the same method. Note also that the equations reported below both for shock and rarefactions waves are strictly valid only if $B^{x} \neq 0$ and indeed should be used only in regions R4 and R5 of the Riemann fan shown in Figure 2 In these regions, only slow-waves are present and these do not appear when $B^{x}=0$.

\subsection{Solution across a shock front}

To calculate the solution across a shock front within the $B_{t}$-method we start by considering the same system of equations in Section 4.1 but we solve equations (4.1)-4.3 considering $B^{y}$ and $B^{z}$ as the unknown quantities. From equations (4.19) and (4.20) we express the post-shock values of $v^{y}$ and $v^{z}$ :

$$
\begin{aligned}
& v_{b}^{y}=\frac{1}{B^{x}}\left[B_{a}^{y} \frac{J}{W_{s} D_{a}}-B_{b}^{y}\left(\frac{J}{W_{s} D_{a}}+v_{a}^{x}-v_{b}^{x}\right)+B^{x} v_{a}^{y}\right], \\
& v_{b}^{z}=\frac{1}{B^{x}}\left[B_{a}^{z} \frac{J}{W_{s} D_{a}}-B_{b}^{z}\left(\frac{J}{W_{s} D_{a}}+v_{a}^{x}-v_{b}^{x}\right)+B^{x} v_{a}^{z}\right] .
\end{aligned}
$$

Using now equation (4.13) to obtain the post-shock value of $D$

$$
D_{b}=\frac{D_{a} J}{J+D_{a} W_{s}\left(v_{a}^{x}-v_{b}^{x}\right)},
$$

and calculating the post-shock value of the total pressure using the invariance of $h_{g} B_{n}$, i.e. $\left[\left[h_{g} B_{n}\right]\right]=0$ (see Anile 1989), we can express all of the quantities as a function of the postshock values of $v^{x}, B^{y}, B^{z}$, and of the shock-velocity $V_{s}$. An analytic solution for the postshock value of $v^{x}$ in terms of the other post-shock quantities was sought but not found, forcing to the numerical solution of one of the equations 4.15-4.17). Furthermore, in analogy with what done in the $p$-method, we calculate the value of the shock velocity by solving numerically equation 4.26.

Finally, it may be useful to point out that the numerical solution of equation 4.26 is at times complicated by the existence of two acceptable roots in the interval of velocities in which the value of the slow shock velocity has to be found (i.e. between the value of $v^{x}$ and the value of the Alfvèn velocity). Because only one of these two roots will lead to a convergent exact solution, a careful selection needs to be made. The existence of these two roots could be related to a known problem in Newtonian MHD where the use of the tangential components of the magnetic field as the post-shock independent variables can lead to the presence of more than one solution ( $c f$., for instance, Jeffrey and Taniuti 1964). This problem seems to be present also in relativistic MHD (Komissarov 2003), but it has not represented a serious drawback for the approach followed here. More work is needed to determine whether the use of the tangential components of the magnetic field as the post-shock independent variables is really optimal or whether different choices are preferable.

\subsection{Solution across a rarefaction wave}

To calculate the solution across a rarefaction wave within the $B_{t}$-method we use the same set of ODEs (4.29)-4.36) discussed in Section 4.2, with the only but important difference that we do not use $\xi$ as self-similar variable but, rather, the norm of the tangential components of the magnetic field $B_{t}$. More specifically, we use equations (4.29)-4.31) together with equations 
(4.35)-4.36) and substitute the derivative with respect to $\xi$ with the one with respect to $B_{t}$. In addition to these equations, which provide a solution for variables $\rho, p, v^{x}, v^{y}$ and $v^{z}$, we express explicitly the relation between the norm and the tangential components in terms of the angle $\psi$

$$
\begin{gathered}
B^{y}=\cos \psi B_{t}, \\
B^{z}=\sin \psi B_{t},
\end{gathered}
$$

and rewrite them as ODEs having $B_{t}$ as the self-similar variable

$$
\begin{aligned}
& \frac{\mathrm{d} B^{y}}{\mathrm{~d} B_{t}}=\cos \psi, \\
& \frac{\mathrm{d} B^{z}}{\mathrm{~d} B_{t}}=\sin \psi .
\end{aligned}
$$

Note that in deriving equations (5.6)-(5.7), an implicit assumption has been made: i.e. that the angle $\psi$ is constant across the rarefaction wave and thus that the tangential magnetic field does not rotate across the rarefaction wave. With the use of the supplementary equations (5.6)-(5.7), the resulting system of ODEs is complete and can be solved numerically using standard techniques for the solution of a system of coupled ODEs. In practice, the integration is started ahead of the rarefaction and is progressed toward the contact discontinuity, where $B_{t}$ is given by the values of $B^{y}$ and $B^{z}$ chosen at the contact discontinuity. In all of the tests reported here (with the exception of test number 5 of Balsara 2001; see Section 6.2.1 for a discussion), the assumption $\psi=$ const. is valid. This is probably related to the choice of the initial conditions used in these tests and in particular to the fact that $v_{A}^{y}=v_{A}^{z}, B_{A}^{y}=B_{A}^{z}$, or $v_{A}^{z}=B_{A}^{z}=0$, where $A=($ left, right $)$, so that the initial states are essentially invariant after the exchange of $y$ with $z$ or the $z$ components of $v$ and $B$ remain equal to zero in all the regions.

It should be noted that also in relativistic hydrodynamics the velocity components tangential to a nonlinear wave can change their norm across the wave, in contrast with what happens in Newtonian hydrodynamics. Considering for simplicity the case for a shock wave in the limit of zero magnetic field, equations (4.16-4.17 reduce to $\left[\left[S^{y} / D\right]\right]=0=\left[\left[S^{z} / D\right]\right]$, indicating that the ratio $v^{y} / v^{z}$ remains unchanged through shocks so that the tangential velocity 3 -vector can change its norm but does not rotate. This property, which applies also across rarefaction waves, is not present across Newtonian nonlinear waves, in which the tangential 3-velocity vector does not rotate, nor changes its norm: $\left[\left[v^{y}\right]\right]=0=\left[\left[v^{z}\right]\right]$.

Although the condition $\psi=$ const. is exact in nonrelativistic MHD, it may not be valid in relativistic regimes, where the tangential magnetic field is instead free to rotate across the slow rarefaction. In this case, a new strategy needs to be implemented and the simplest one consists of using the angle $\psi$ as the self-similar variable so that the system of equations 4.29-4.36 can be expressed in terms of this new integration variable. In addition, the supplementary differential equation for one of the components of the tangential magnetic field can be obtained through the algebraic relation

$$
B^{y}=\frac{\cos \psi}{\sin \psi} B^{z}
$$

and its derivative with respect to $\psi$

$$
\frac{\mathrm{d} B^{y}}{\mathrm{~d} \psi}=\frac{\cos \psi}{\sin \psi} \frac{\mathrm{d} B^{z}}{\mathrm{~d} \psi}-\frac{1}{\sin ^{2} \psi} B^{z} .
$$

The integration of the system of ODEs is done starting from the value of $\psi$ given by the ratio of the tangential components of the magnetic field ahead of the rarefaction wave, up to the value 
given by the amplitudes of $B^{y}$ and $B^{z}$ at the contact discontinuity. Furthermore, as for the $p$ method, also within the $B_{t}$-method the values of the variable $\xi$ are obtained from the quartic equation 4.50.

A representative example of this effect is shown in Figure 13 where we plot the exact solution of the generic Alfvèn test at time $t=1.5$ ( $c f$. Table 4 for the initial conditions of this test). In particular, the left panel of Figure 13 shows the norm of the tangential magnetic field $B_{t}$, while the right panel the angle $\psi \equiv \arctan \left(B^{z} / B^{y}\right)$. Note how both quantities vary across all the fast, slow and Alfvèn waves.

\section{Numerical Implementation and Representative Results}

Since the properties of the magnetic field components in the initial states lead to considerably different Riemann problems ( $c f$. the two Riemann fans in Figures 11 and 2), we will discuss separately the numerical solution in the cases in which $B^{x}=0$ and $B^{x} \neq 0$, emphasizing the properties of some of the most representative tests.

\subsection{Tangential Initial Magnetic Field: $B^{x}=0$}

As discussed in Section 3 , when $B^{x}=0$ the Riemann problem consists of only two fast-waves and of a tangential discontinuity across which only $v^{x}$ and $p$ are continuous ( $c f$. Figure 1). It should be noted that the condition of continuity of the total pressure across the tangential discontinuity does not necessarily extend also to the gas pressure and, indeed, the latter is in general discontinuous ( $c f$. Figures 3 and 4). In essence, the numerical solution of the Riemann problem when $B^{x}=0$ proceeds as follows: given the initial left and right states (i.e. regions R1 and R4 of Figure 1, we follow the procedure used in relativistic hydrodynamics and determine two unknown states as function of the common total pressure in regions R2 and R3 ( $p$-method). The jump in the normal component of the velocity at the tangential discontinuity is then checked and a new guess for the total pressure found. This procedure is iterated until the solution is found with the desired accuracy. The numerical approach used is a combination of Newton-Raphson and bisection methods, starting from a value for the total pressure which is the average of the initial left and right states. Furthermore, to decide whether the wave considered is a shock or a rarefaction, we compare the values of the total pressure ahead of and behind the wave, solving the set of equations across a shock if the guessed value is larger than the total pressure ahead of the wave and thus in the initial state. We note that this procedure could be improved if an approach similar to the one discussed by Rezzolla et al. $(2001,2003)$ is implemented, which would exploit the values of the initial relative velocity to predict the wave-pattern produced.

It is also worth noting that even though the numerical strategy discussed so far is very similar to the one used in relativistic hydrodynamics, the equations to be solved in MHD are much more complex and, more importantly, their computational cost markedly larger. This is essentially because an analytic expression for the shock velocity was not found, so that the latter must be determined numerically.

\subsubsection{Representative Tests for $B^{x}=0$}

Because initial states with a zero normal magnetic field lead to a Riemann problem that is comparatively much simpler to solve, an independent numerical code has been built for this case and it has been tested to reproduce known results in relativistic hydrodynamics, as well as a test proposed by Komissarov (1999) (this is referred to as the "shock-tube" test 2). We have also considered an additional, more generic shock-tube test in which all of the quantities in the initial states are nonzero and in which $\vec{v} \cdot \vec{B} \neq 0$ (this is referred to as the "generic shock-tube" test) $\dagger$.

$\dagger$ We note that a Riemann problem with $B^{x}=0$, but with $\vec{v} \cdot \vec{B} \neq 0$ cannot be solved with the exact solution recently proposed by Romero et al. (2005). 


\begin{tabular}{|c|c|c|c|c|c|c|c|c|}
\hline Test type & $\rho$ & $p_{\mathrm{g}}$ & $v^{x}$ & $v^{y}$ & $v^{z}$ & $B^{x}$ & $B^{y}$ & $B^{z}$ \\
\hline Komissarov: Shock-Tube $2 \begin{array}{l}(\Gamma=4 / 3) \\
\text { left state } \\
\text { right state }\end{array}$ & $\begin{array}{l}1.0 \\
0.1\end{array}$ & $\begin{array}{c}30.0 \\
1.0\end{array}$ & $\begin{array}{l}0.0 \\
0.0\end{array}$ & $\begin{array}{l}0.0 \\
0.0\end{array}$ & $\begin{array}{l}0.0 \\
0.0\end{array}$ & $\begin{array}{l}0.0 \\
0.0\end{array}$ & $\begin{array}{c}20.0 \\
0.0\end{array}$ & $\begin{array}{l}0.0 \\
0.0\end{array}$ \\
\hline $\begin{aligned} & \text { Generic Shock-Tube }(\Gamma=5 / 3) \\
& \text { left state } \\
& \text { right state }\end{aligned}$ & $\begin{array}{c}1.0 \\
0.01\end{array}$ & $\begin{array}{c}0.01 \\
5000\end{array}$ & $\begin{array}{l}0.1 \\
0.5\end{array}$ & $\begin{array}{l}0.3 \\
0.4\end{array}$ & $\begin{array}{l}0.4 \\
0.3\end{array}$ & $\begin{array}{l}0.0 \\
0.0\end{array}$ & $\begin{array}{l}6.0 \\
5.0\end{array}$ & $\begin{array}{c}2.0 \\
20.0\end{array}$ \\
\hline
\end{tabular}

TABLE 1. Initial conditions for the tests of the exact Riemann solver when the magnetic field has zero normal component, i.e. $B^{x}=0$.

\begin{tabular}{|c|c|c|c|c|c|c|c|}
\hline & $\rho$ & $p$ & $v^{x}$ & $v^{y}$ & $v^{z}$ & $B^{y}$ & $B^{z}$ \\
\hline $\mathrm{R} 1$ & $0.1000 \mathrm{E}+01$ & $0.2300 \mathrm{E}+03$ & $0.0000 \mathrm{E}+00$ & $0.0000 \mathrm{E}+00$ & $0.0000 \mathrm{E}+00$ & $0.2000 \mathrm{E}+02$ & $0.0000 \mathrm{E}+00$ \\
$\mathrm{R} 2$ & $0.2410 \mathrm{E}+00$ & $0.1611 \mathrm{E}+02$ & $0.8497 \mathrm{E}+00$ & $0.0000 \mathrm{E}+00$ & $0.0000 \mathrm{E}+00$ & $0.9141 \mathrm{E}+01$ & $0.0000 \mathrm{E}+00$ \\
$\mathrm{R} 3$ & $0.6426 \mathrm{E}+00$ & $0.1611 \mathrm{E}+02$ & $0.8497 \mathrm{E}+00$ & $0.0000 \mathrm{E}+00$ & $0.0000 \mathrm{E}+00$ & $0.0000 \mathrm{E}+00$ & $0.0000 \mathrm{E}+00$ \\
$\mathrm{R} 4$ & $0.1000 \mathrm{E}+00$ & $0.1000 \mathrm{E}+01$ & $0.0000 \mathrm{E}+00$ & $0.0000 \mathrm{E}+00$ & $0.0000 \mathrm{E}+00$ & $0.0000 \mathrm{E}+00$ & $0.0000 \mathrm{E}+00$ \\
\hline
\end{tabular}

TABLE 2. First significant digits for the exact solution of the test shock-tube 2 of Komissarov (1999) computed with an accuracy of $10^{-12}$. The left column indicates the regions in which the solution is computed (cf. Fig. 11.

\begin{tabular}{|c|c|c|c|c|c|c|c|}
\hline & $\rho$ & $p$ & $v^{x}$ & $v^{y}$ & $v^{z}$ & $B^{y}$ & $B^{z}$ \\
\hline $\mathrm{R} 1$ & $0.1000 \mathrm{E}+01$ & $0.1819 \mathrm{E}+02$ & $0.1000 \mathrm{E}+00$ & $0.3000 \mathrm{E}+00$ & $0.4000 \mathrm{E}+00$ & $0.6000 \mathrm{E}+01$ & $0.2000 \mathrm{E}+01$ \\
$\mathrm{R} 2$ & $0.1581 \mathrm{E}+01$ & $0.4459 \mathrm{E}+02$ & $-0.3073 \mathrm{E}+00$ & $0.3082 \mathrm{E}+00$ & $0.2927 \mathrm{E}+00$ & $0.9582 \mathrm{E}+01$ & $0.3194 \mathrm{E}+01$ \\
$\mathrm{R} 3$ & $0.5489 \mathrm{E}-03$ & $0.4459 \mathrm{E}+02$ & $-0.3073 \mathrm{E}+00$ & $0.7488 \mathrm{E}+00$ & $0.5556 \mathrm{E}+00$ & $0.1023 \mathrm{E}+01$ & $0.4092 \mathrm{E}+01$ \\
$\mathrm{R} 4$ & $0.1000 \mathrm{E}-01$ & $0.5138 \mathrm{E}+04$ & $0.5000 \mathrm{E}+00$ & $0.4000 \mathrm{E}+00$ & $0.3000 \mathrm{E}+00$ & $0.5000 \mathrm{E}+01$ & $0.2000 \mathrm{E}+02$ \\
\hline
\end{tabular}

TABLE 3. The same as Table 2 but for the generic shock-tube test computed with an accuracy of $10^{-11}$.

Because the procedure for calculating the solution in this case is particularly simple and well tested from relativistic hydrodynamics, the algorithm employed has shown to be very robust and no failures were encountered in the calculation of any quantity. We list in Table1 the set of initial conditions used in the tests solved, while we report in Tables 2 and 3 the first significant digits for the exact solution of the same tests, reporting in all cases the accuracy obtained (which usually is $\lesssim 10^{-11}$ ). Finally, the full solutions in space of the various Riemann problems listed in Table 1 and for the quantities $\rho, v^{x}, p_{\mathrm{g}}, p, v^{y}, v^{z}, B^{y}$, and $B^{z}$ are shown in Figures 3 and 4 at the indicated representative times. 

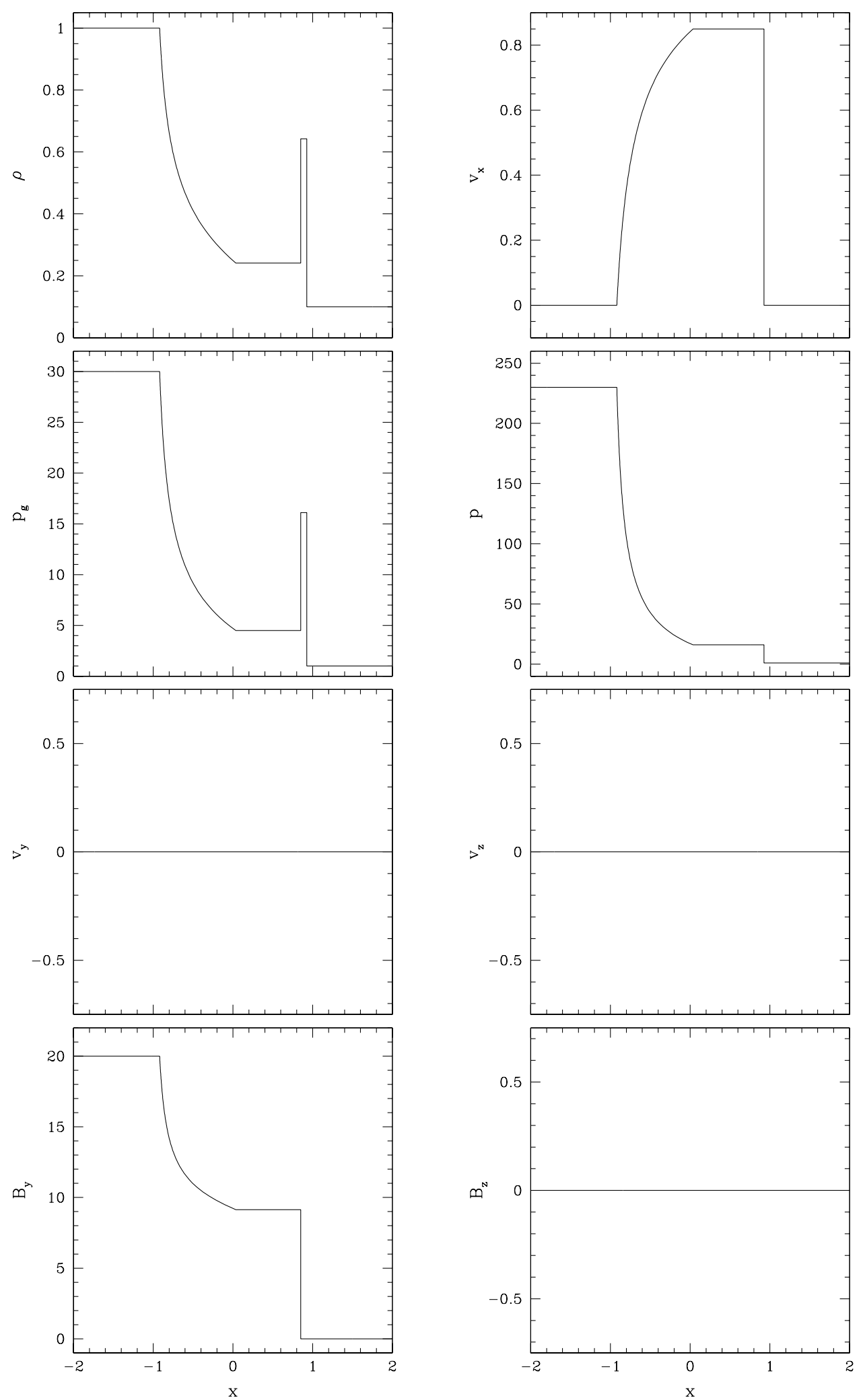

FIGURE 3. Exact solution of the test shock-tube 2 of Komissarov (1999) at time $t=1.0$. The solution is composed of a left-going rarefaction wave, a tangential discontinuity and a right-going shock. 

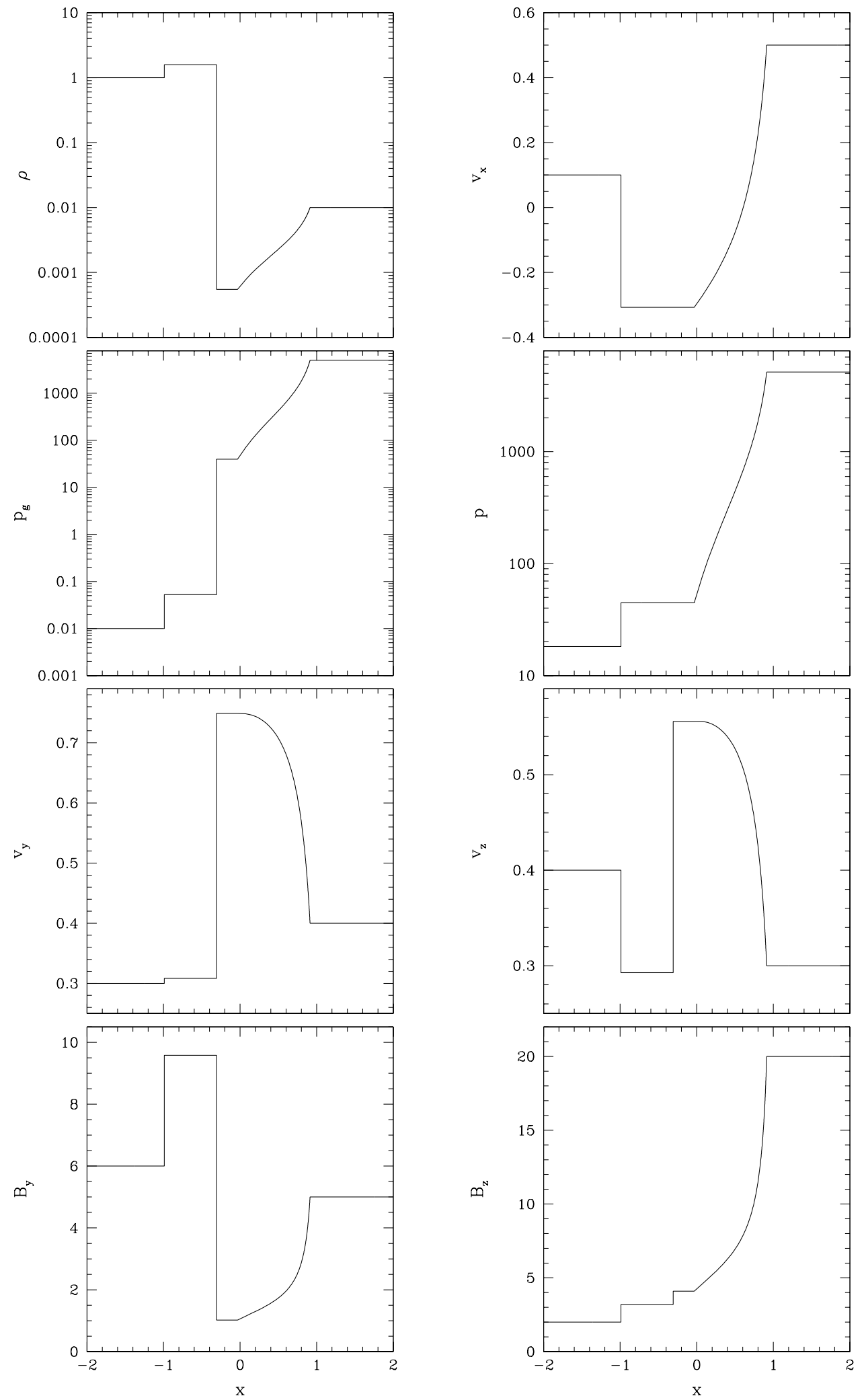

FIGURE 4. Exact solution of the generic shock-tube test at time $t=1.0$. The solution is composed of a left-going shock, a tangential discontinuity and a right-going rarefaction wave 


\subsection{Generic Initial Magnetic field: $B^{x} \neq 0$}

As discussed in Section 3 when $B^{x} \neq 0$ the Riemann problem consists of seven different waves: two fast-waves, two slow-waves, two Alfvèn discontinuities and a central contact discontinuity across which only the density can be discontinuous ( $c f$. Figure [2]. In essence, the numerical solution of the Riemann problem when $B^{x} \neq 0$ proceeds as follows: starting from the initial left and right states (i.e. regions R1 and R8 of Figure 2), we compute the states after the fast-waves (regions R2 and R7), then we determine the jumps across the Alfvèn discontinuities (regions R3 and R6) and finally we solve the equations for the slow-waves (regions R4 and R5). As a result of this sequence, the jump conditions in all the physical variables in the two states across the contact discontinuity are computed and if the solution obtained in this way does not reach the desired accuracy, the procedure is iterated.

We also recall that when $B^{x} \neq 0$, the numerical solution is found using a hybrid method which adopts different sets of equations according to the region in which the Riemann problem has to be solved. In particular, to compute the states after the fast-waves and across the Alfvèn discontinuities we use as unknown the total pressure ( $p$-method; Section (4) and to discriminate between shocks and rarefaction waves we evaluate the jump in the total pressure in a way similar to the case when $B^{x}=0$. To compute the states after the slow-waves, on the other hand, we use the tangential components of the magnetic field ( $B_{t}$-method; Section 5) and to decide whether a wave is a shock or a rarefaction we evaluate the jump in the norm of the magnetic field bearing in mind that it must decrease across slow shocks and increase otherwise. Then at the contact discontinuity we compute the jumps in the total pressure and in the components of three-velocity and if they are above a certain accuracy we iterate by changing the values of the total pressure, used in regions R2-R3 and R6-R7, and of the tangential components of the magnetic field, used in regions $\mathrm{R} 4-\mathrm{R} 5$.

It is worth underlining that the solution of the Riemann problem with generic initial states is considerably more demanding than when $B^{x}=0$ and not only because of the more numerous waves present. Indeed, the most severe difficulty is due to the fact that the set of equations to be solved becomes particularly stiff near the solution. A careful investigation of the several cases considered has in fact revealed that, in general, the functional behavior of the quantities whose roots are sought, changes very rapidly near the roots, stretching the ability of standards rootfinding algorithms. As a result, it is not uncommon that the solution cannot be found if the iteration for the search of the root starts from a guess which is not sufficiently close to the exact solution. To avoid such failures and to provide a first guess which is reasonably accurate, we have used as a guide the solution provided by the HLLE approximate Riemann solver†. In practice, the approximate solution should be accurate to a few percent in the regions away from the waves, where the states are almost constant (very close to the waves the errors are of course much larger). Using this guess has proven to be sufficient to obtain a solution in all of the cases considered, but of course there is no guarantee that a solution will be straightforwardly found for all of the possible initial states. Our experience when the solution could not be immediately obtained is that an increase in the accuracy of the approximate Riemann solver is in general sufficient to yield a convergent and accurate solution.

\subsubsection{Representative Tests for $B^{x} \neq 0$}

Although the numerical code developed for the exact solution of the Riemann problem in relativistic MHD could in principle be used for generic initial data, we have used it in particular to calculate the exact solutions of those less trivial initial states that over the years have become standard references (e.g. Komissarov 1999, or Balsara 2001). Table 4 collects the set of initial

$\dagger$ Note that this is not necessary when $B^{x}=0$ since in this case the solution can also be quite far from the exact one and yet the iterative scheme does not show problems in converging to it. 


\begin{tabular}{|c|c|c|c|c|c|c|c|c|}
\hline Test type & $\rho$ & $p_{\mathrm{g}}$ & $v^{x}$ & $v^{y}$ & $v^{z}$ & $B^{x}$ & $B^{y}$ & $B^{z}$ \\
\hline $\begin{array}{c}\text { Komissarov: Shock-Tube Test } 1 \quad \begin{array}{l}(\Gamma=4 / 3) \\
\text { left state } \\
\text { right state }\end{array} \\
\end{array}$ & $\begin{array}{l}1.0 \\
0.1 \\
\end{array}$ & $\begin{array}{c}1000.0 \\
1.0\end{array}$ & $\begin{array}{l}0.0 \\
0.0\end{array}$ & $\begin{array}{l}0.0 \\
0.0 \\
\end{array}$ & $\begin{array}{l}0.0 \\
0.0 \\
\end{array}$ & $\begin{array}{l}1.0 \\
1.0\end{array}$ & $\begin{array}{l}0.0 \\
0.0\end{array}$ & $\begin{array}{l}0.0 \\
0.0 \\
\end{array}$ \\
\hline $\begin{aligned} & \text { Komissarov: Collision Test }(\Gamma=4 / 3) \\
& \text { left state } \\
& \text { right state }\end{aligned}$ & $\begin{array}{l}1.0 \\
1.0\end{array}$ & $\begin{array}{l}1.0 \\
1.0\end{array}$ & $\begin{array}{c}5 / \sqrt{26} \\
-5 / \sqrt{26}\end{array}$ & $\begin{array}{l}0.0 \\
0.0\end{array}$ & $\begin{array}{l}0.0 \\
0.0\end{array}$ & $\begin{array}{l}10.0 \\
10.0\end{array}$ & $\begin{array}{r}10.0 \\
-10.0\end{array}$ & $\begin{array}{l}0.0 \\
0.0\end{array}$ \\
\hline $\begin{array}{r}\text { Balsara Test } 1 \text { (Brio \& Wu) }(\Gamma=2) \\
\text { left state } \\
\text { right state }\end{array}$ & $\begin{array}{l}1.000 \\
0.125 \\
\end{array}$ & $\begin{array}{l}1.0 \\
0.1 \\
\end{array}$ & $\begin{array}{l}0.0 \\
0.0 \\
\end{array}$ & $\begin{array}{l}0.0 \\
0.0 \\
\end{array}$ & $\begin{array}{l}0.0 \\
0.0 \\
\end{array}$ & $\begin{array}{l}0.5 \\
0.5 \\
\end{array}$ & $\begin{array}{r}1.0 \\
-1.0 \\
\end{array}$ & $\begin{array}{l}0.0 \\
0.0 \\
\end{array}$ \\
\hline 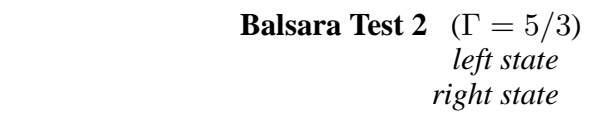 & $\begin{array}{l}1.0 \\
1.0\end{array}$ & $\begin{array}{c}30.0 \\
1.0\end{array}$ & $\begin{array}{l}0.0 \\
0.0\end{array}$ & $\begin{array}{l}0.0 \\
0.0\end{array}$ & $\begin{array}{l}0.0 \\
0.0\end{array}$ & $\begin{array}{l}5.0 \\
5.0\end{array}$ & $\begin{array}{l}6.0 \\
0.7\end{array}$ & $\begin{array}{l}6.0 \\
0.7\end{array}$ \\
\hline $\begin{array}{c}\text { Balsara Test } 3 \quad(\Gamma=5 / 3) \\
\text { left state } \\
\text { right state }\end{array}$ & $\begin{array}{l}1.0 \\
1.0\end{array}$ & $\begin{array}{c}1000.0 \\
0.1\end{array}$ & $\begin{array}{l}0.0 \\
0.0\end{array}$ & $\begin{array}{l}0.0 \\
0.0\end{array}$ & $\begin{array}{l}0.0 \\
0.0\end{array}$ & $\begin{array}{l}10.0 \\
10.0\end{array}$ & $\begin{array}{l}7.0 \\
0.7\end{array}$ & $\begin{array}{l}7.0 \\
0.7\end{array}$ \\
\hline $\begin{array}{c}\text { Balsara Test } 4 \quad(\Gamma=5 / 3) \\
\text { left state } \\
\text { right state }\end{array}$ & $\begin{array}{l}1.0 \\
1.0\end{array}$ & $\begin{array}{l}0.1 \\
0.1\end{array}$ & $\begin{array}{c}0.999 \\
-0.999\end{array}$ & $\begin{array}{l}0.0 \\
0.0\end{array}$ & $\begin{array}{l}0.0 \\
0.0\end{array}$ & $\begin{array}{l}10.0 \\
10.0\end{array}$ & $\begin{array}{c}7.0 \\
-7.0\end{array}$ & $\begin{array}{r}7.0 \\
-7.0\end{array}$ \\
\hline $\begin{array}{c}\text { Balsara Test } 5 \quad(\Gamma=5 / 3) \\
\text { left state } \\
\text { right state }\end{array}$ & $\begin{array}{l}1.08 \\
1.00\end{array}$ & $\begin{array}{c}0.95 \\
1.0\end{array}$ & $\begin{array}{r}0.40 \\
-0.45\end{array}$ & $\begin{array}{c}0.3 \\
-0.2\end{array}$ & $\begin{array}{l}0.2 \\
0.2\end{array}$ & $\begin{array}{l}2.0 \\
2.0\end{array}$ & $\begin{array}{c}0.3 \\
-0.7\end{array}$ & $\begin{array}{l}0.3 \\
0.5\end{array}$ \\
\hline $\begin{array}{c}\text { Generic Alfvèn Test } \begin{array}{l}(\Gamma=5 / 3) \\
\text { left state } \\
\text { right state }\end{array}\end{array}$ & $\begin{array}{l}1.0 \\
0.9\end{array}$ & $\begin{array}{l}5.0 \\
5.3\end{array}$ & $\begin{array}{l}0.0 \\
0.0\end{array}$ & $\begin{array}{l}0.3 \\
0.0\end{array}$ & $\begin{array}{l}0.4 \\
0.0\end{array}$ & $\begin{array}{l}1.0 \\
1.0\end{array}$ & $\begin{array}{l}6.0 \\
5.0\end{array}$ & $\begin{array}{l}2.0 \\
2.0\end{array}$ \\
\hline
\end{tabular}

TABLE 4. Initial conditions for the tests of the exact Riemann solver when the magnetic field has nonzero normal component, i.e. $B^{x} \neq 0$.

conditions used in the tests solved, while we report in Tables 5 - 12 the first significant digits for the exact solution of the same tests, reporting in all cases the accuracy obtained (which usually is $\sim 10^{-10}$ ). Finally, the full solutions in space of the various Riemann problems listed in Table 4 and for the quantities $\rho, v^{x}, p_{\mathrm{g}}, p, v^{y}, v^{z}, B^{y}$, and $B^{z}$ are shown in Figures 5 -12 at the indicated representative times. In addition, Figure 13 offers a quantitative view of the changes in the tangential magnetic field $B_{t}$ and of the rotation angle $\psi$ across the fast, slow and Alfvèn waves in the case of a generic Alfvèn test.

In all of the tests reported in Table 4 the HLLE solver with about 800 gridpoints was able to track rather well the exact solution in all of its waves. The only exception to this has been test number 1 of Balsara (2001) which represents the relativistic version of the test proposed by Brio-Wu (1988) in Newtonian hydrodynamics (van Putten 1993). The approximate numerical solution of this test, in fact, shows the development of a left-going slow compound-wave, that is a wave composed by a slow shock adjacent to a slow rarefaction. Since we assume that a slow or fast-wave can either be a pure rarefaction or a pure shock, compound structures of this type 


\begin{tabular}{|c|c|c|c|c|c|c|c|}
\hline & $\rho$ & $p$ & $v^{x}$ & $v^{y}$ & $v^{z}$ & $B^{y}$ & $B^{z}$ \\
\hline R1 & $0.1000 \mathrm{E}+01$ & $0.1001 \mathrm{E}+04$ & $0.0000 \mathrm{E}+00$ & $0.0000 \mathrm{E}+00$ & $0.0000 \mathrm{E}+00$ & $0.0000 \mathrm{E}+00$ & $0.0000 \mathrm{E}+00$ \\
R2 & $0.6984 \mathrm{E}-01$ & $0.2927 \mathrm{E}+02$ & $0.9115 \mathrm{E}+00$ & $0.0000 \mathrm{E}+00$ & $0.0000 \mathrm{E}+00$ & $0.0000 \mathrm{E}+00$ & $0.0000 \mathrm{E}+00$ \\
R3 & $0.6984 \mathrm{E}-01$ & $0.2927 \mathrm{E}+02$ & $0.9115 \mathrm{E}+00$ & $0.0000 \mathrm{E}+00$ & $0.0000 \mathrm{E}+00$ & $0.0000 \mathrm{E}+00$ & $0.0000 \mathrm{E}+00$ \\
R4 & $0.6984 \mathrm{E}-01$ & $0.2927 \mathrm{E}+02$ & $0.9115 \mathrm{E}+00$ & $0.0000 \mathrm{E}+00$ & $0.0000 \mathrm{E}+00$ & $0.0000 \mathrm{E}+00$ & $0.0000 \mathrm{E}+00$ \\
R5 & $0.8846 \mathrm{E}+00$ & $0.2927 \mathrm{E}+02$ & $0.9115 \mathrm{E}+00$ & $0.0000 \mathrm{E}+00$ & $0.0000 \mathrm{E}+00$ & $0.0000 \mathrm{E}+00$ & $0.0000 \mathrm{E}+00$ \\
R6 & $0.8846 \mathrm{E}+00$ & $0.2927 \mathrm{E}+02$ & $0.9115 \mathrm{E}+00$ & $0.0000 \mathrm{E}+00$ & $0.0000 \mathrm{E}+00$ & $0.0000 \mathrm{E}+00$ & $0.0000 \mathrm{E}+00$ \\
R7 & $0.8846 \mathrm{E}+00$ & $0.2927 \mathrm{E}+02$ & $0.9115 \mathrm{E}+00$ & $0.0000 \mathrm{E}+00$ & $0.0000 \mathrm{E}+00$ & $0.0000 \mathrm{E}+00$ & $0.0000 \mathrm{E}+00$ \\
R8 & $0.1000 \mathrm{E}+00$ & $0.1500 \mathrm{E}+01$ & $0.0000 \mathrm{E}+00$ & $0.0000 \mathrm{E}+00$ & $0.0000 \mathrm{E}+00$ & $0.0000 \mathrm{E}+00$ & $0.0000 \mathrm{E}+00$ \\
\hline
\end{tabular}

TABLE 5. First significant digits for the exact solution of the test shock-tube 1 of Komissarov (1999) computed with an accuracy of $10^{-10}$. The left column indicates the regions in which the solution is computed (cf. Fig. 2].

cannot be found by construction and thus are not present in the exact solution found (cf. Table 7 and Figure 77. We remark that it is not yet clear whether compound waves have to be considered acceptable physical solutions of the ideal MHD equations and a debate on this is still ongoing (see, for instance, Myong \& Roe 1997a Myong \& Roe 1997b Falle \& Komissarov 2001 Torrilhon 2003a Torrilhon 2003b Torrilhon \& Balsara 2004). We here prefer to adopt the same standpoint of Ryu and Jones (1995) in the development of their exact Riemann solver in nonrelativistic magnetohydrodynamics and not comment further on this until a commonly accepted view has emerged.

Another test which deserves a special comment is test number 5 of Balsara (2001), in which the left-going Alfvèn discontinuity and the left-going slow rarefaction wave have very similar propagation velocities. Indeed they are so close to each other that not even the HLLE approximate Riemann solver with 40000 gridpoints was able to capture the precise location of the discontinuity. As a consequence, the initial guess for the jumps across the left-going Alfvèn discontinuity was sufficiently good to yield a convergent solution, but not good enough to provide an exact solution with a truncation error comparable with the one reached in all of the other tests ( $c f$. data in Table 11). In addition, another distinctive feature of this test and which has not been found in any of the others, is the rotation of the angle $\psi$ across the left-going slow rarefaction. To handle this we have followed the procedure discussed in Section 5.2] and used equation (5.9) to compute the changes in the tangential magnetic field across the rarefaction wave.

\section{Conclusions}

We have presented the procedure for the solution of the exact Riemann problem in special relativistic MHD. Special care has been paid in treating both degenerate initial states (i.e. with zero normal magnetic field) leading to a set of only three waves analogous to the ones in relativistic hydrodynamics, as well as generic initial states (i.e. with nonzero normal magnetic field) leading to the full set of seven MHD waves.

The approach discussed for the numerical solution of the exact Riemann problem reflects this distinction and different sets of equations are used according to the values of the normal magnetic field. In particular, when $B^{x}=0$, all of the equations needed for the solution of the Riemann problem are written as a function of the total pressure, thus following a procedure which is logically equivalent to the one adopted in relativistic hydrodynamics (we have referred to this as to the $p$-method). When $B^{x} \neq 0$, on the other hand, an hybrid approach is adopted in which the 

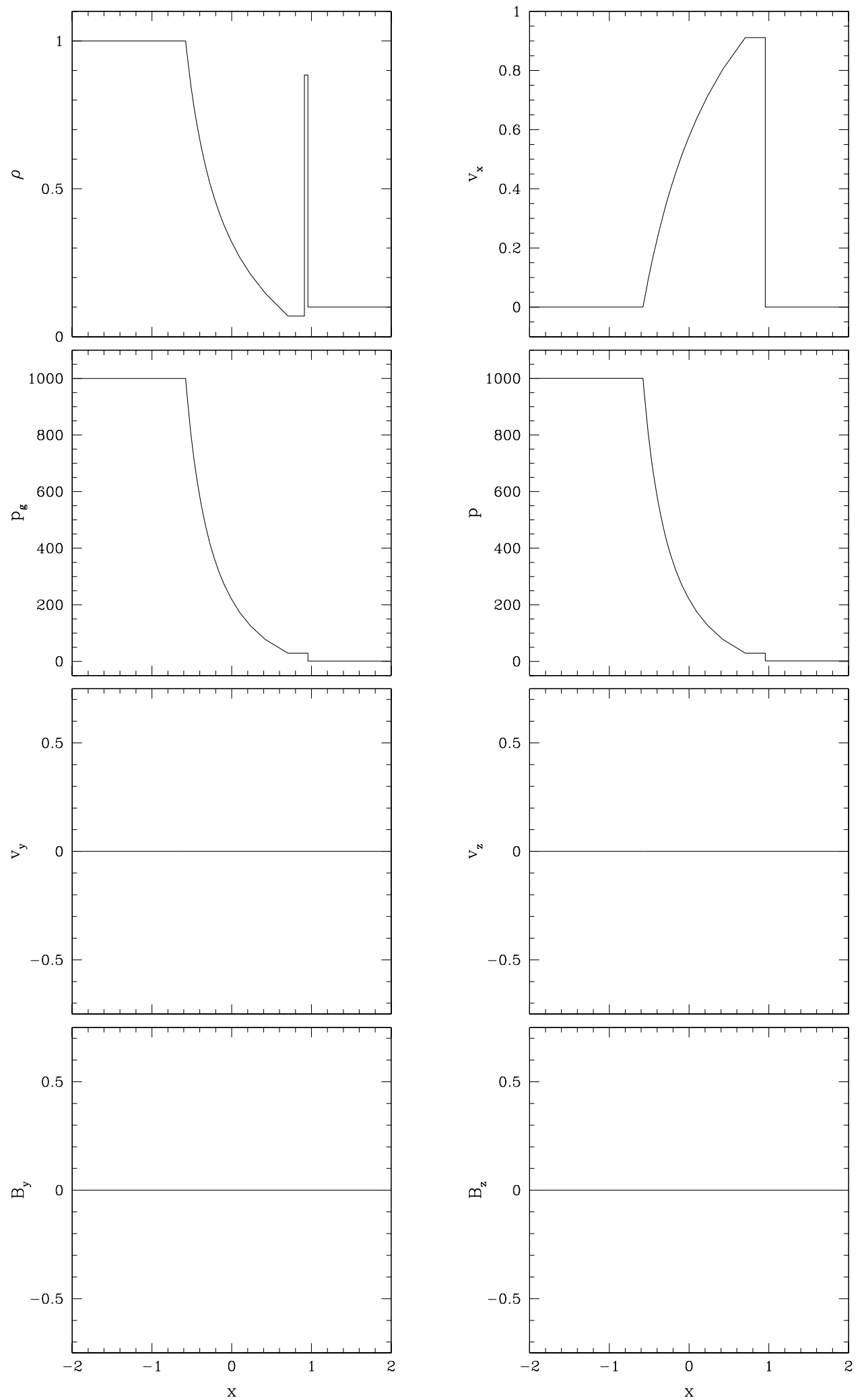

FIGURE 5. Exact solution of the test shock-tube 1 of Komissarov (1999) at time $t=1.0$. The solution is composed of a left-going fast rarefaction, of a contact discontinuity and of a right-going fast shock. 

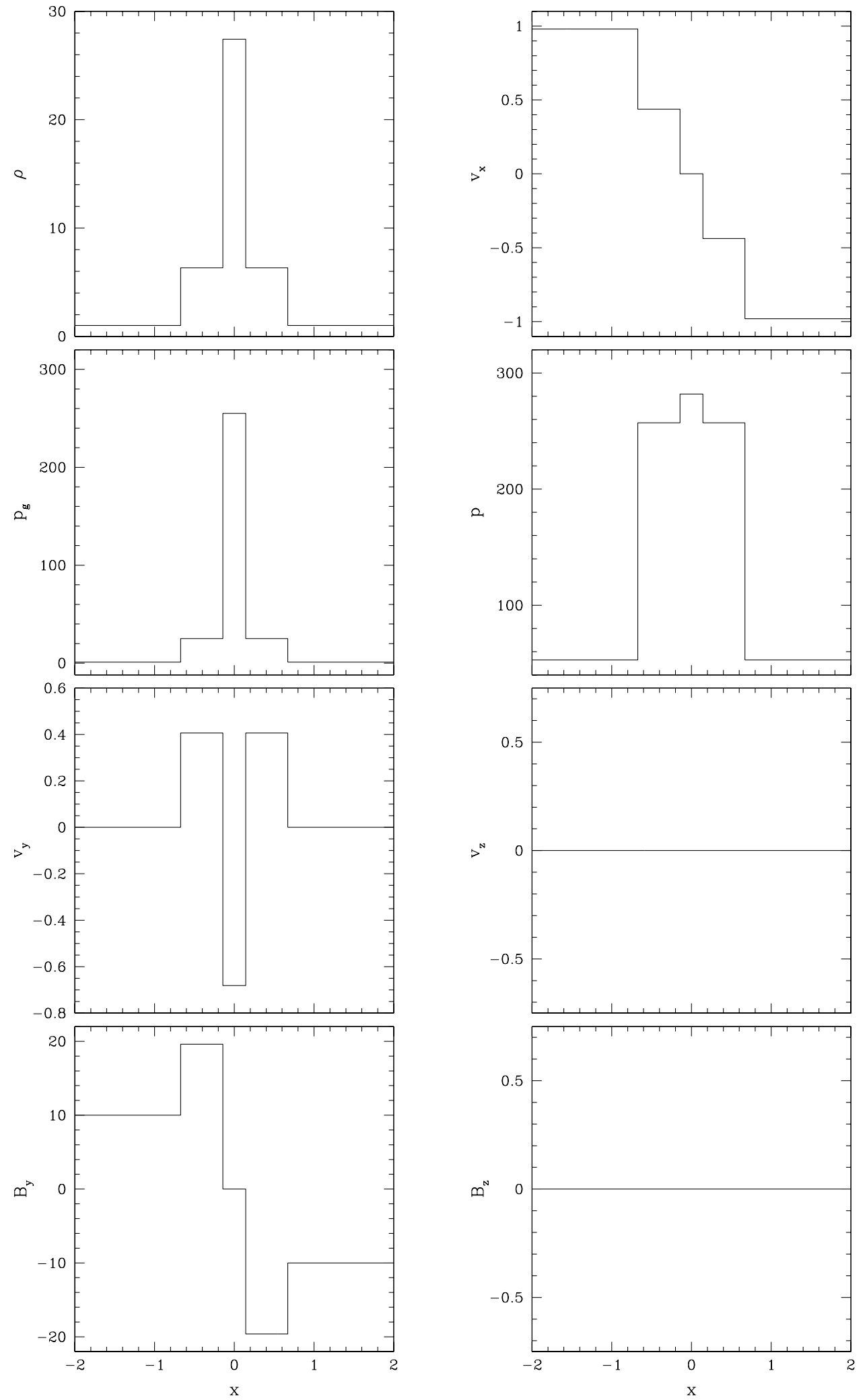

FIGURE 6. Exact solution of the collision test of Komissarov (1999) at time $t=1.22$. The solution is composed of a left-going fast shock, of a left-going slow shock, a right-going slow shock and of a right-going fast shock. 

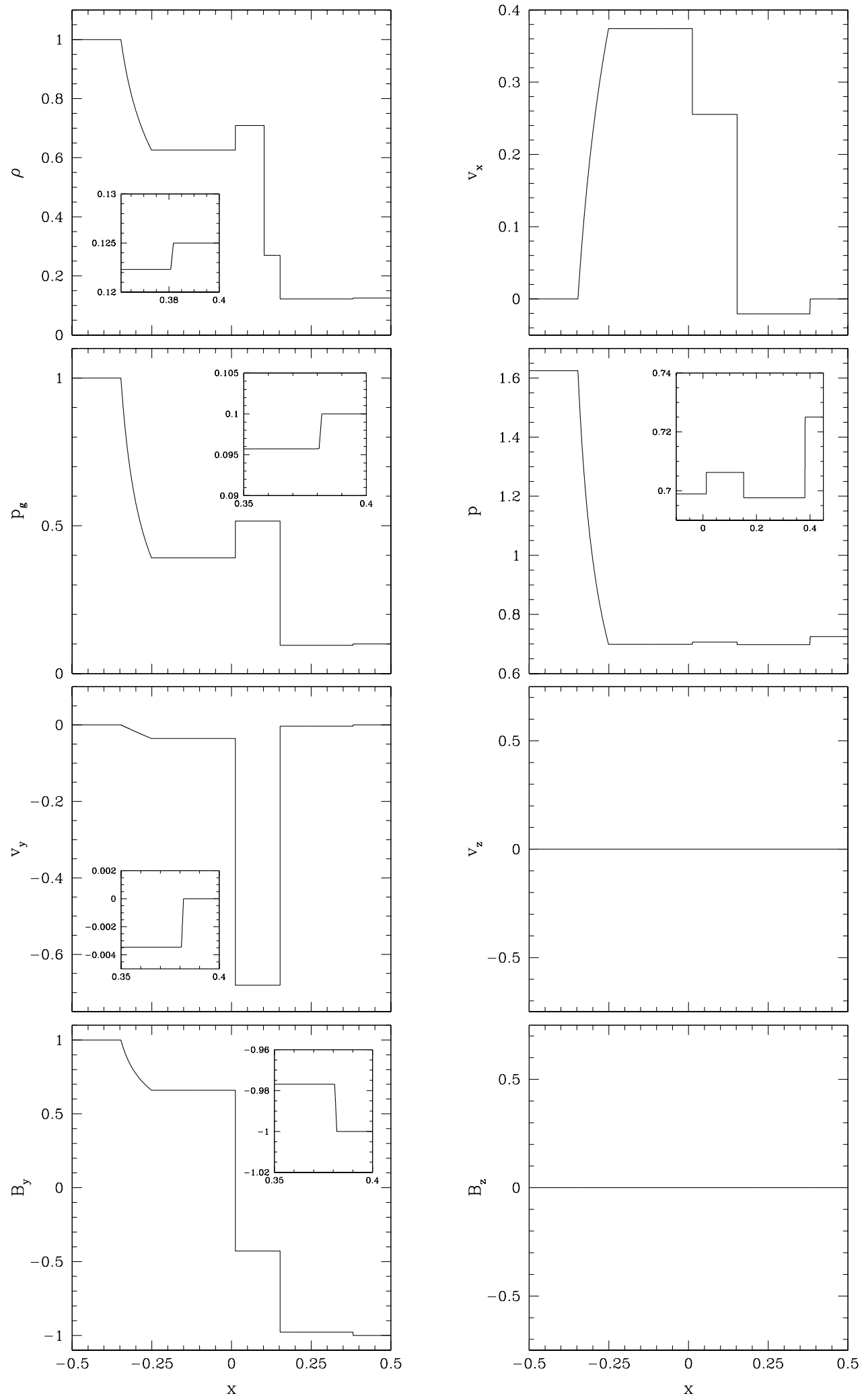

FIGURE 7. Exact solution of the test number 1 of Balsara (2001) at time $t=0.4$ and which represents the relativistic version of the Brio-Wu test (1988). The solution is composed of a left-going fast rarefaction, of a left-going slow shock, of a contact discontinuity, of a right-going slow shock and of a right-going fast rarefaction. Note the absence of a slow compound-wave which cannot be found by construction in our exact solver, but that appears in the solution of the HLLE approximate Riemann solver (not shown). 

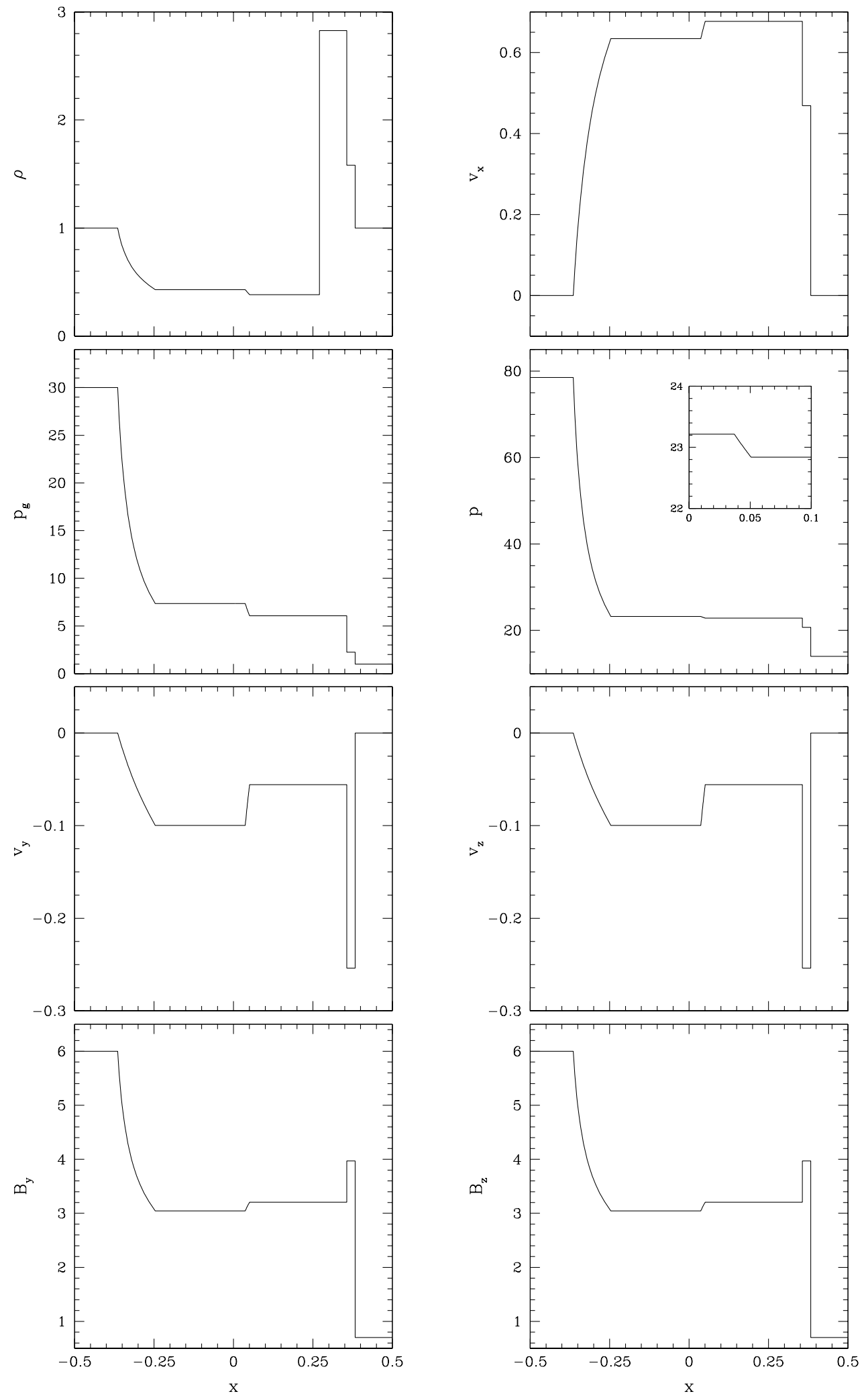

FIGURE 8. Exact solution of the test number 2 of Balsara (2001) at time $t=0.4$. The solution is composed of two left-going fast and slow rarefactions, of a contact discontinuity and of two right-going fast and slow shocks. 

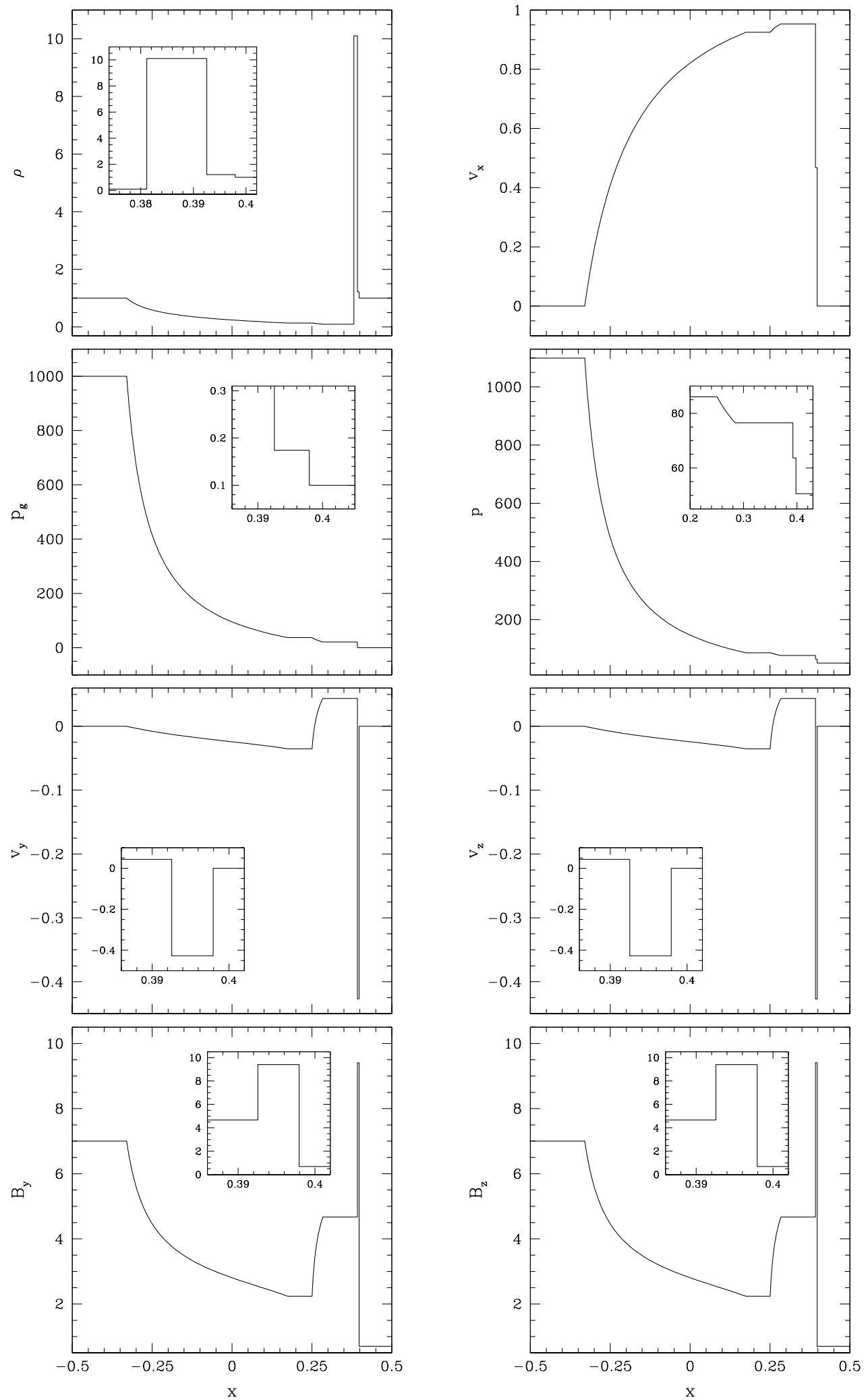

FIGURE 9. Exact solution of the test number 3 of Balsara (2001) at time $t=0.4$. The solution is composed of two left-going fast and slow rarefactions, of a contact discontinuity and of two right-going fast and slow shocks. 

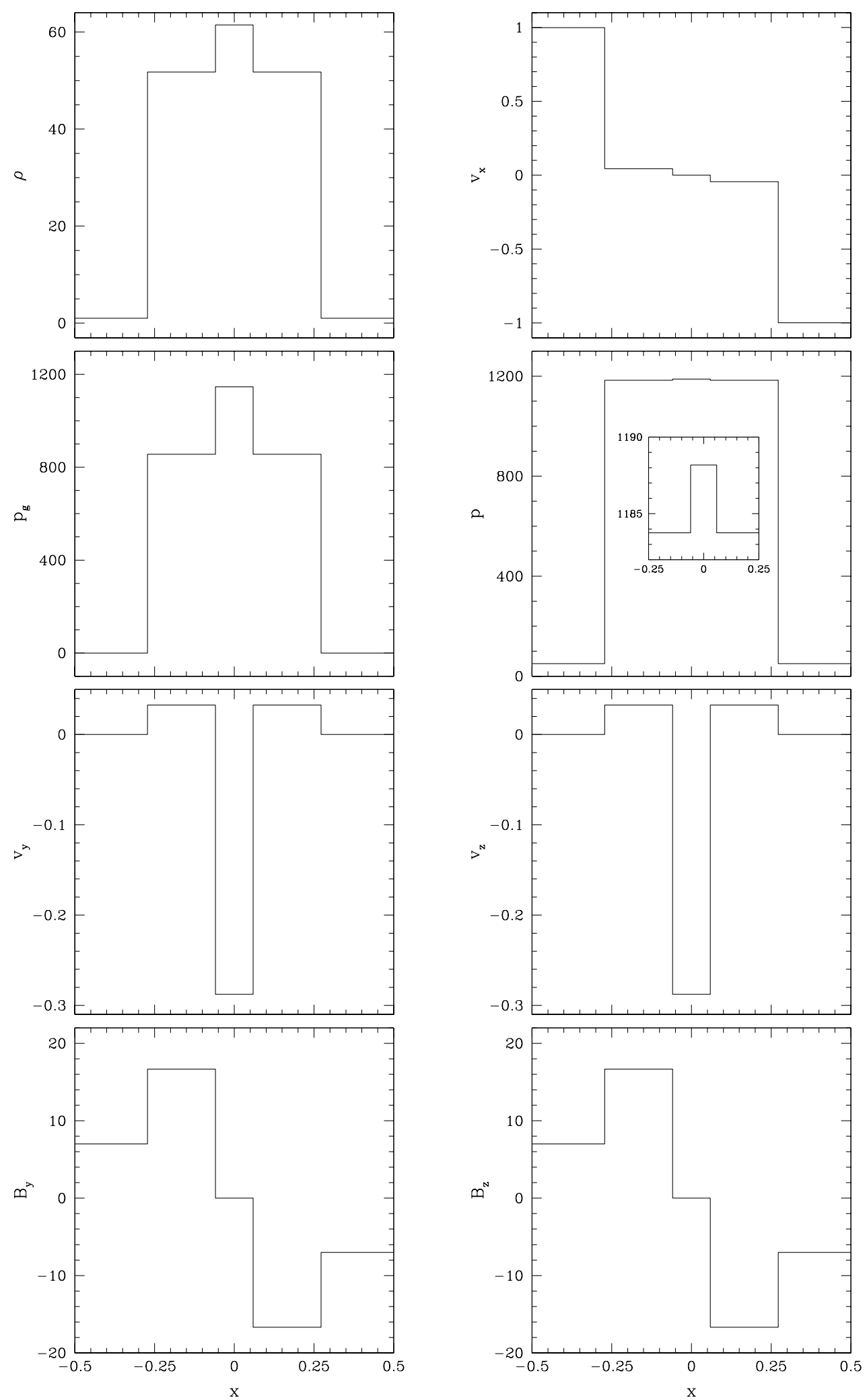

FIGURE 10. Exact solution of the test number 4 of Balsara (2001) at time $t=0.4$. The solution is composed of two left-going fast and slow shocks and of two right-going fast and slow shocks. 

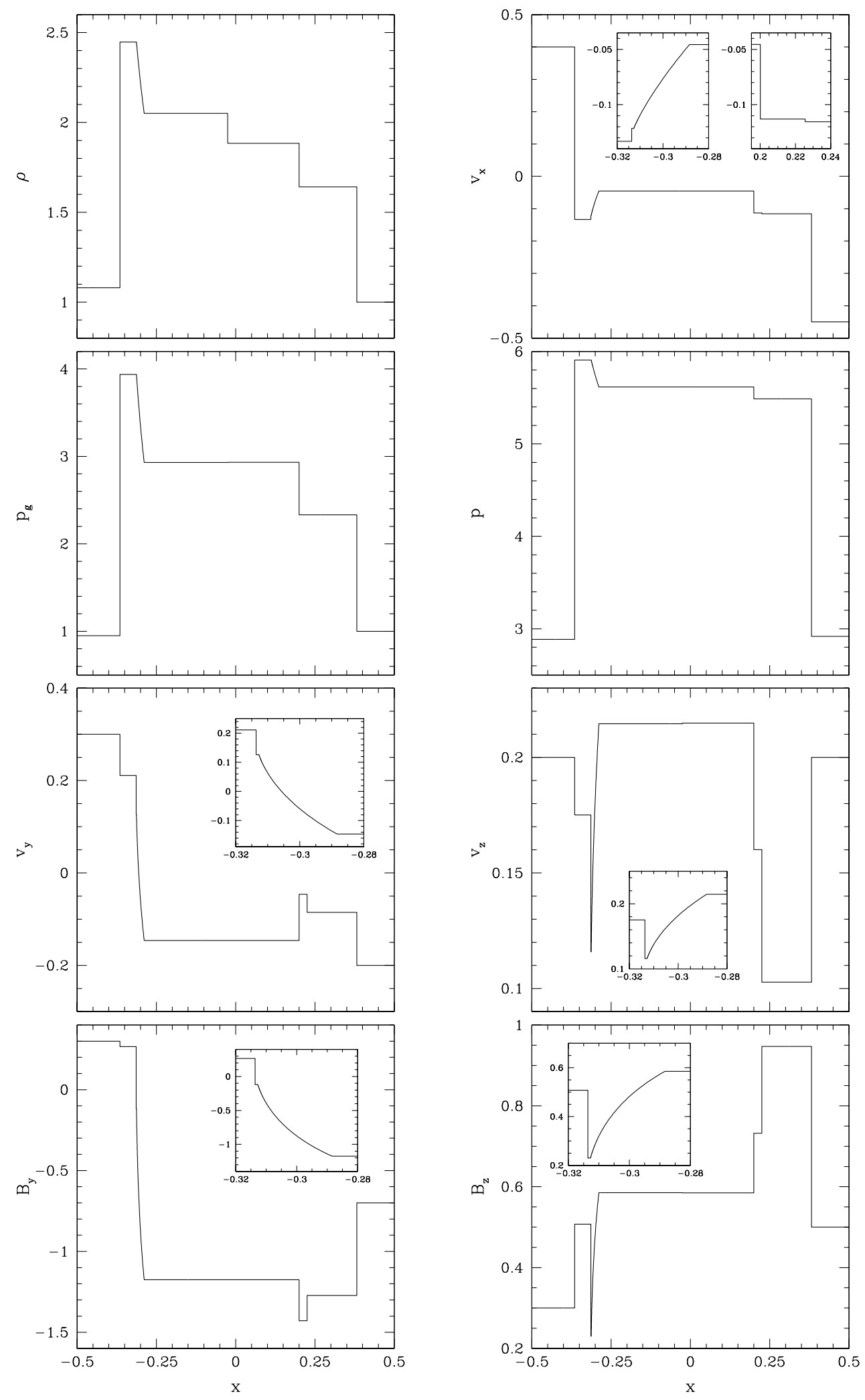

FIGURE 11. Exact solution of the test number 5 of Balsara (2001) at time $t=0.55$. The solution is composed of a left-going fast shock, of a left-going Alfvèn discontinuity, of a left-going slow rarefaction, of a contact discontinuity, of a right-going slow shock, of a right-going Alfvèn discontinuity and of a right-going fast shock. Note that the accuracy in this test is only rather low: $3 \times 10^{-4}$. 

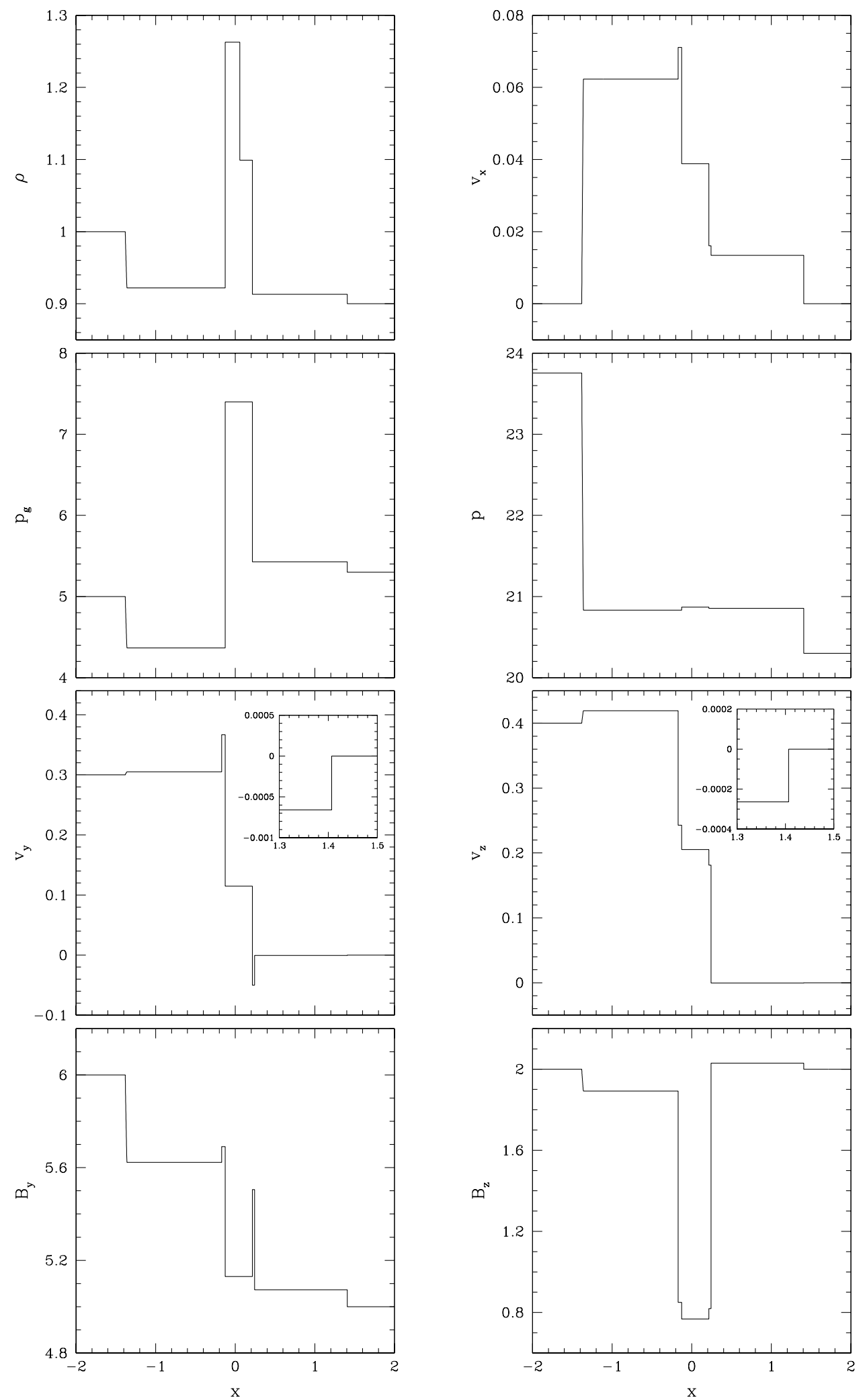

FIGURE 12. Exact solution of the generic Alfvèn test at time $t=1.5$. The solution is composed of a left-going fast rarefaction, of a left-going Alfvèn discontinuity, of a left-going slow shock, of a contact discontinuity, of a right-going slow shock, of a right-going Alfvèn discontinuity and of a right-going fast shock. 

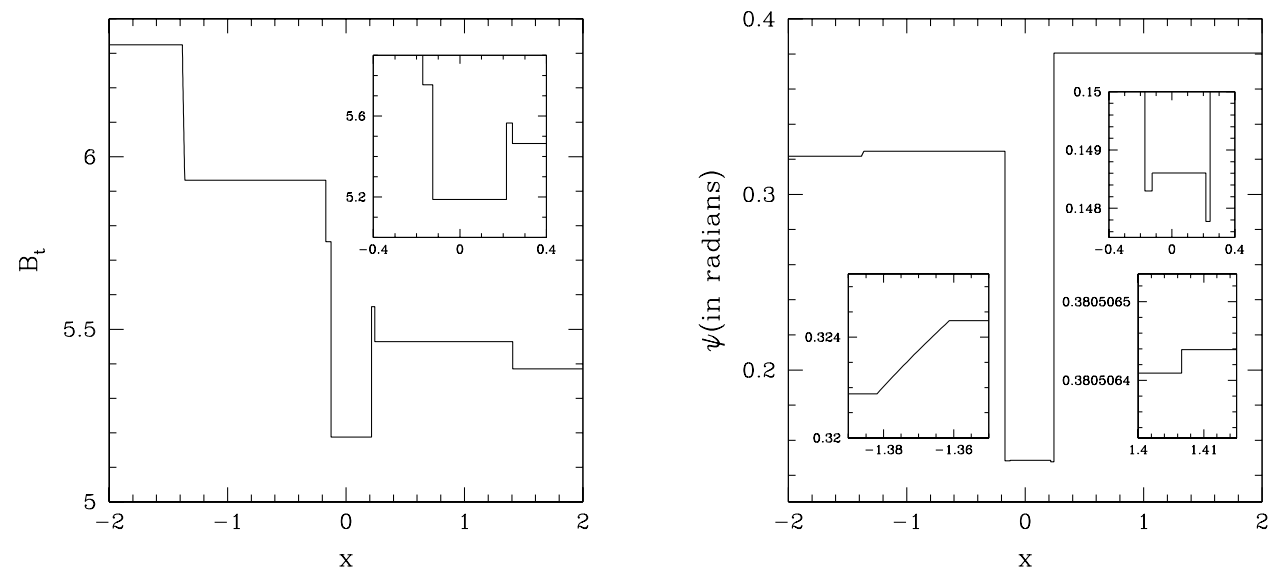

FIGURE 13. Exact solution of the generic Alfvèn test at time $t=1.5$. The left panel shows the norm of the tangential magnetic field $B_{t}$, while the right panel the angle $\psi \equiv \arctan \left(B^{z} / B^{y}\right)$. Note that both quantities vary across all the fast, slow and Alfvèn waves as a result of a relativistic effect.

\begin{tabular}{|c|c|c|c|c|c|c|c|}
\hline & $\rho$ & $p$ & $v^{x}$ & $v^{y}$ & $v^{z}$ & $B^{y}$ & $B^{z}$ \\
\hline R1 & $0.1000 \mathrm{E}+01$ & $0.5292 \mathrm{E}+02$ & $0.9806 \mathrm{E}+00$ & $0.0000 \mathrm{E}+00$ & $0.0000 \mathrm{E}+00$ & $0.1000 \mathrm{E}+02$ & $0.0000 \mathrm{E}+00$ \\
R2 & $0.6331 \mathrm{E}+01$ & $0.2571 \mathrm{E}+03$ & $0.4380 \mathrm{E}+00$ & $0.4069 \mathrm{E}+00$ & $0.0000 \mathrm{E}+00$ & $0.1960 \mathrm{E}+02$ & $0.0000 \mathrm{E}+00$ \\
R3 & $0.6331 \mathrm{E}+01$ & $0.2571 \mathrm{E}+03$ & $0.4380 \mathrm{E}+00$ & $0.4069 \mathrm{E}+00$ & $0.0000 \mathrm{E}+00$ & $0.1960 \mathrm{E}+02$ & $0.0000 \mathrm{E}+00$ \\
R4 & $0.2742 \mathrm{E}+02$ & $0.2819 \mathrm{E}+03$ & $0.2453 \mathrm{E}-07$ & $-0.6811 \mathrm{E}+00$ & $0.0000 \mathrm{E}+00$ & $0.2250 \mathrm{E}-06$ & $0.0000 \mathrm{E}+00$ \\
R5 & $0.2742 \mathrm{E}+02$ & $0.2819 \mathrm{E}+03$ & $-0.2810 \mathrm{E}-07$ & $-0.6811 \mathrm{E}+00$ & $0.0000 \mathrm{E}+00$ & $0.2250 \mathrm{E}-06$ & $0.0000 \mathrm{E}+00$ \\
R6 & $0.6331 \mathrm{E}+01$ & $0.2571 \mathrm{E}+03$ & $-0.4380 \mathrm{E}+00$ & $0.4069 \mathrm{E}+00$ & $0.0000 \mathrm{E}+00$ & $-0.1960 \mathrm{E}+02$ & $0.0000 \mathrm{E}+00$ \\
R7 & $0.6331 \mathrm{E}+01$ & $0.2571 \mathrm{E}+03$ & $-0.4380 \mathrm{E}+00$ & $0.4069 \mathrm{E}+00$ & $0.0000 \mathrm{E}+00$ & $-0.1960 \mathrm{E}+02$ & $0.0000 \mathrm{E}+00$ \\
R8 & $0.1000 \mathrm{E}+01$ & $0.5292 \mathrm{E}+02$ & $-0.9806 \mathrm{E}+00$ & $0.0000 \mathrm{E}+00$ & $0.0000 \mathrm{E}+00$ & $-0.1000 \mathrm{E}+02$ & $0.0000 \mathrm{E}+00$ \\
\hline
\end{tabular}

TABLE 6. The same as Table 5 but for the exact solution of the test Collision of Komissarov (1999) computed with an accuracy of $10^{-6}$.

\begin{tabular}{|c|c|c|c|c|c|c|c|}
\hline & $\rho$ & $p$ & $v^{x}$ & $v^{y}$ & $v^{z}$ & $B^{y}$ & $B^{z}$ \\
\hline R1 & $0.1000 \mathrm{E}+01$ & $0.1625 \mathrm{E}+01$ & $0.0000 \mathrm{E}+00$ & $0.0000 \mathrm{E}+00$ & $0.0000 \mathrm{E}+00$ & $0.1000 \mathrm{E}+01$ & $0.0000 \mathrm{E}+00$ \\
R2 & $0.6257 \mathrm{E}+00$ & $0.6989 \mathrm{E}+00$ & $0.3742 \mathrm{E}+00$ & $-0.3561 \mathrm{E}-01$ & $0.0000 \mathrm{E}+00$ & $0.6594 \mathrm{E}+00$ & $0.0000 \mathrm{E}+00$ \\
R3 & $0.6257 \mathrm{E}+00$ & $0.6989 \mathrm{E}+00$ & $0.3742 \mathrm{E}+00$ & $-0.3561 \mathrm{E}-01$ & $0.0000 \mathrm{E}+00$ & $0.6594 \mathrm{E}+00$ & $0.0000 \mathrm{E}+00$ \\
R4 & $0.7092 \mathrm{E}+00$ & $0.7062 \mathrm{E}+00$ & $0.2555 \mathrm{E}+00$ & $-0.6804 \mathrm{E}+00$ & $0.0000 \mathrm{E}+00$ & $-0.4285 \mathrm{E}+00$ & $0.0000 \mathrm{E}+00$ \\
R5 & $0.2695 \mathrm{E}+00$ & $0.7062 \mathrm{E}+00$ & $0.2555 \mathrm{E}+00$ & $-0.6804 \mathrm{E}+00$ & $0.0000 \mathrm{E}+00$ & $-0.4285 \mathrm{E}+00$ & $0.0000 \mathrm{E}+00$ \\
R6 & $0.1223 \mathrm{E}+00$ & $0.6976 \mathrm{E}+00$ & $-0.2080 \mathrm{E}-01$ & $-0.3460 \mathrm{E}-02$ & $0.0000 \mathrm{E}+00$ & $-0.9769 \mathrm{E}+00$ & $0.0000 \mathrm{E}+00$ \\
R7 & $0.1223 \mathrm{E}+00$ & $0.6976 \mathrm{E}+00$ & $-0.2080 \mathrm{E}-01$ & $-0.3460 \mathrm{E}-02$ & $0.0000 \mathrm{E}+00$ & $-0.9769 \mathrm{E}+00$ & $0.0000 \mathrm{E}+00$ \\
R8 & $0.1250 \mathrm{E}+00$ & $0.7250 \mathrm{E}+00$ & $0.0000 \mathrm{E}+00$ & $0.0000 \mathrm{E}+00$ & $0.0000 \mathrm{E}+00$ & $-0.1000 \mathrm{E}+01$ & $0.0000 \mathrm{E}+00$ \\
\hline
\end{tabular}

TABLE 7. The same as Table 5 but for the exact solution of the test number 1 of Balsara (2001) computed with an accuracy of $10^{-10}$. This test represents the relativistic version of the test proposed by Brio \& Wu (1988) 


\begin{tabular}{|c|c|c|c|c|c|c|c|}
\hline & $\rho$ & $p$ & $v^{x}$ & $v^{y}$ & $v^{z}$ & $B^{y}$ & $B^{z}$ \\
\hline R1 & $0.1000 \mathrm{E}+01$ & $0.7850 \mathrm{E}+02$ & $0.0000 \mathrm{E}+00$ & $0.0000 \mathrm{E}+00$ & $0.0000 \mathrm{E}+00$ & $0.6000 \mathrm{E}+01$ & $0.6000 \mathrm{E}+01$ \\
R2 & $0.4300 \mathrm{E}+00$ & $0.2321 \mathrm{E}+02$ & $0.6344 \mathrm{E}+00$ & $-0.9981 \mathrm{E}-01$ & $-0.9981 \mathrm{E}-01$ & $0.3045 \mathrm{E}+01$ & $0.3045 \mathrm{E}+01$ \\
R3 & $0.4300 \mathrm{E}+00$ & $0.2321 \mathrm{E}+02$ & $0.6344 \mathrm{E}+00$ & $-0.9981 \mathrm{E}-01$ & $-0.9981 \mathrm{E}-01$ & $0.3045 \mathrm{E}+01$ & $0.3045 \mathrm{E}+01$ \\
R4 & $0.3830 \mathrm{E}+00$ & $0.2284 \mathrm{E}+02$ & $0.6770 \mathrm{E}+00$ & $-0.5566 \mathrm{E}-01$ & $-0.5566 \mathrm{E}-01$ & $0.3205 \mathrm{E}+01$ & $0.3205 \mathrm{E}+01$ \\
R5 & $0.2828 \mathrm{E}+01$ & $0.2284 \mathrm{E}+02$ & $0.6770 \mathrm{E}+00$ & $-0.5566 \mathrm{E}-01$ & $-0.5566 \mathrm{E}-01$ & $0.3205 \mathrm{E}+01$ & $0.3205 \mathrm{E}+01$ \\
R6 & $0.1582 \mathrm{E}+01$ & $0.2072 \mathrm{E}+02$ & $0.4688 \mathrm{E}+00$ & $-0.2538 \mathrm{E}+00$ & $-0.2538 \mathrm{E}+00$ & $0.3971 \mathrm{E}+01$ & $0.3971 \mathrm{E}+01$ \\
R7 & $0.1582 \mathrm{E}+01$ & $0.2072 \mathrm{E}+02$ & $0.4688 \mathrm{E}+00$ & $-0.2538 \mathrm{E}+00$ & $-0.2538 \mathrm{E}+00$ & $0.3971 \mathrm{E}+01$ & $0.3971 \mathrm{E}+01$ \\
R8 & $0.1000 \mathrm{E}+01$ & $0.1399 \mathrm{E}+02$ & $0.0000 \mathrm{E}+00$ & $0.0000 \mathrm{E}+00$ & $0.0000 \mathrm{E}+00$ & $0.7000 \mathrm{E}+00$ & $0.7000 \mathrm{E}+00$ \\
\hline
\end{tabular}

TABLE 8. The same as Table 5 but for the exact solution of the test number 2 of Balsara (2001) computed with an accuracy of $10^{-10}$.

\begin{tabular}{|c|c|c|c|c|c|c|c|}
\hline & $\rho$ & $p$ & $v^{x}$ & $v^{y}$ & $v^{z}$ & $B^{y}$ & $B^{z}$ \\
\hline R1 & $0.1000 \mathrm{E}+01$ & $0.1099 \mathrm{E}+04$ & $0.0000 \mathrm{E}+00$ & $0.0000 \mathrm{E}+00$ & $0.0000 \mathrm{E}+00$ & $0.7000 \mathrm{E}+01$ & $0.7000 \mathrm{E}+01$ \\
R2 & $0.1381 \mathrm{E}+00$ & $0.8604 \mathrm{E}+02$ & $0.9246 \mathrm{E}+00$ & $-0.3513 \mathrm{E}-01$ & $-0.3513 \mathrm{E}-01$ & $0.2238 \mathrm{E}+01$ & $0.2238 \mathrm{E}+01$ \\
R3 & $0.1381 \mathrm{E}+00$ & $0.8604 \mathrm{E}+02$ & $0.9246 \mathrm{E}+00$ & $-0.3513 \mathrm{E}-01$ & $-0.3513 \mathrm{E}-01$ & $0.2238 \mathrm{E}+01$ & $0.2238 \mathrm{E}+01$ \\
R4 & $0.9798 \mathrm{E}-01$ & $0.7653 \mathrm{E}+02$ & $0.9529 \mathrm{E}+00$ & $0.4366 \mathrm{E}-01$ & $0.4366 \mathrm{E}-01$ & $0.4670 \mathrm{E}+01$ & $0.4670 \mathrm{E}+01$ \\
R5 & $0.1010 \mathrm{E}+02$ & $0.7653 \mathrm{E}+02$ & $0.9529 \mathrm{E}+00$ & $0.4366 \mathrm{E}-01$ & $0.4366 \mathrm{E}-01$ & $0.4670 \mathrm{E}+01$ & $0.4670 \mathrm{E}+01$ \\
R6 & $0.1218 \mathrm{E}+01$ & $0.6363 \mathrm{E}+02$ & $0.4670 \mathrm{E}+00$ & $-0.4270 \mathrm{E}+00$ & $-0.4270 \mathrm{E}+00$ & $0.9408 \mathrm{E}+01$ & $0.9408 \mathrm{E}+01$ \\
R7 & $0.1218 \mathrm{E}+01$ & $0.6363 \mathrm{E}+02$ & $0.4670 \mathrm{E}+00$ & $-0.4270 \mathrm{E}+00$ & $-0.4270 \mathrm{E}+00$ & $0.9408 \mathrm{E}+01$ & $0.9408 \mathrm{E}+01$ \\
R8 & $0.1000 \mathrm{E}+01$ & $0.5059 \mathrm{E}+02$ & $0.0000 \mathrm{E}+00$ & $0.0000 \mathrm{E}+00$ & $0.0000 \mathrm{E}+00$ & $0.7000 \mathrm{E}+00$ & $0.7000 \mathrm{E}+00$ \\
\hline
\end{tabular}

TABLE 9. The same as Table 5 but for the exact solution of the test number 3 of Balsara (2001) computed with an accuracy of $10^{-10}$.

solution across fast-waves and Alfvèn discontinuities is still computed using the $p$-method, but the one across slow-waves and the contact discontinuity is computed using equations which are written in terms of the tangential components of the magnetic field (we have referred to this as to the $B_{t}$-method). The use of a combined approach for the general case of $B^{x} \neq 0$ has turned out to be crucial for a successful solution of the problem.

Because of its generality, the solution presented here could serve as a useful if not indispensable test for those numerical codes that solve the MHD equations in relativistic regimes. As the astronomical observations become increasingly more accurate, such numerical codes will become increasingly more important to explain and describe in detail the complex physics of astrophysical compact objects.

As a final remark we note that despite the considerable improvements in the performance of modern computers, the exact solution of the Riemann problem at each grid interface is still computationally too expensive to be used routinely in sophisticated multidimensional numerical codes solving the equations of relativistic hydrodynamics or MHD in either stationary or dynamical spacetimes (see, for instance, Baiotti et al. 2005, Duez et al. 2005). While a numerical code based on exact Riemann solvers may represent at least in principle the most accurate approach to the solution of the hydrodynamics and MHD equations, considerable work is still required to make it competitive with less accurate but more computationally efficient methods. A first step 


\begin{tabular}{|c|c|c|c|c|c|c|c|}
\hline & $\rho$ & $p$ & $v^{x}$ & $v^{y}$ & $v^{z}$ & $B^{y}$ & $B^{z}$ \\
\hline R1 & $0.1000 \mathrm{E}+01$ & $0.5020 \mathrm{E}+02$ & $0.9990 \mathrm{E}+00$ & $0.0000 \mathrm{E}+00$ & $0.0000 \mathrm{E}+00$ & $0.7000 \mathrm{E}+01$ & $0.7000 \mathrm{E}+01$ \\
R2 & $0.5175 \mathrm{E}+02$ & $0.1184 \mathrm{E}+04$ & $0.4408 \mathrm{E}-01$ & $0.3263 \mathrm{E}-01$ & $0.3263 \mathrm{E}-01$ & $0.1668 \mathrm{E}+02$ & $0.1668 \mathrm{E}+02$ \\
R3 & $0.5175 \mathrm{E}+02$ & $0.1184 \mathrm{E}+04$ & $0.4408 \mathrm{E}-01$ & $0.3263 \mathrm{E}-01$ & $0.3263 \mathrm{E}-01$ & $0.1668 \mathrm{E}+02$ & $0.1668 \mathrm{E}+02$ \\
R4 & $0.6148 \mathrm{E}+02$ & $0.1188 \mathrm{E}+04$ & $0.1086 \mathrm{E}-07$ & $-0.2877 \mathrm{E}+00$ & $-0.2877 \mathrm{E}+00$ & $0.8042 \mathrm{E}-09$ & $0.8036 \mathrm{E}-09$ \\
R5 & $0.6148 \mathrm{E}+02$ & $0.1188 \mathrm{E}+04$ & $-0.1089 \mathrm{E}-07$ & $-0.2877 \mathrm{E}+00$ & $-0.2877 \mathrm{E}+00$ & $0.8042 \mathrm{E}-09$ & $0.8036 \mathrm{E}-09$ \\
R6 & $0.5175 \mathrm{E}+02$ & $0.1184 \mathrm{E}+04$ & $-0.4408 \mathrm{E}-01$ & $0.3263 \mathrm{E}-01$ & $0.3263 \mathrm{E}-01$ & $-0.1668 \mathrm{E}+02$ & $-0.1668 \mathrm{E}+02$ \\
R7 & $0.5175 \mathrm{E}+02$ & $0.1184 \mathrm{E}+04$ & $-0.4408 \mathrm{E}-01$ & $0.3263 \mathrm{E}-01$ & $0.3263 \mathrm{E}-01$ & $-0.1668 \mathrm{E}+02$ & $-0.1668 \mathrm{E}+02$ \\
R8 & $0.1000 \mathrm{E}+01$ & $0.5020 \mathrm{E}+02$ & $-0.9990 \mathrm{E}+00$ & $0.0000 \mathrm{E}+00$ & $0.0000 \mathrm{E}+00$ & $-0.7000 \mathrm{E}+01$ & $-0.7000 \mathrm{E}+01$ \\
\hline
\end{tabular}

TABLE 10. The same as Table 5 but for the exact solution of the test number 4 of Balsara (2001) computed with an accuracy of $10^{-7}$.

\begin{tabular}{|c|c|c|c|c|c|c|c|}
\hline & $\rho$ & $p$ & $v^{x}$ & $v^{y}$ & $v^{z}$ & $B^{y}$ & $B^{z}$ \\
\hline $\mathrm{R} 1$ & $0.1080 \mathrm{E}+01$ & $0.2885 \mathrm{E}+01$ & $0.4000 \mathrm{E}+00$ & $0.3000 \mathrm{E}+00$ & $0.2000 \mathrm{E}+00$ & $0.3000 \mathrm{E}+00$ & $0.3000 \mathrm{E}+00$ \\
$\mathrm{R} 2$ & $0.2447 \mathrm{E}+01$ & $0.5908 \mathrm{E}+01$ & $-0.1331 \mathrm{E}+00$ & $0.2111 \mathrm{E}+00$ & $0.1751 \mathrm{E}+00$ & $0.2662 \mathrm{E}+00$ & $0.5076 \mathrm{E}+00$ \\
$\mathrm{R} 3$ & $0.2447 \mathrm{E}+01$ & $0.5908 \mathrm{E}+01$ & $-0.1215 \mathrm{E}+00$ & $0.1264 \mathrm{E}+00$ & $0.1158 \mathrm{E}+00$ & $-0.1182 \mathrm{E}+00$ & $0.2302 \mathrm{E}+00$ \\
R4 & $0.2050 \mathrm{E}+01$ & $0.5616 \mathrm{E}+01$ & $-0.4547 \mathrm{E}-01$ & $-0.1463 \mathrm{E}+00$ & $0.2146 \mathrm{E}+00$ & $-0.1175 \mathrm{E}+01$ & $0.5852 \mathrm{E}+00$ \\
R5 & $0.1884 \mathrm{E}+01$ & $0.5616 \mathrm{E}+01$ & $-0.4543 \mathrm{E}-01$ & $-0.1462 \mathrm{E}+00$ & $0.2149 \mathrm{E}+00$ & $-0.1175 \mathrm{E}+01$ & $0.5850 \mathrm{E}+00$ \\
R6 & $0.1642 \mathrm{E}+01$ & $0.5488 \mathrm{E}+01$ & $-0.1129 \mathrm{E}+00$ & $-0.4606 \mathrm{E}-01$ & $0.1601 \mathrm{E}+00$ & $-0.1429 \mathrm{E}+01$ & $0.7320 \mathrm{E}+00$ \\
R7 & $0.1642 \mathrm{E}+01$ & $0.5488 \mathrm{E}+01$ & $-0.1155 \mathrm{E}+00$ & $-0.8536 \mathrm{E}-01$ & $0.1027 \mathrm{E}+00$ & $-0.1272 \mathrm{E}+01$ & $0.9468 \mathrm{E}+00$ \\
R8 & $0.1000 \mathrm{E}+01$ & $0.2918 \mathrm{E}+01$ & $-0.4500 \mathrm{E}+00$ & $-0.2000 \mathrm{E}+00$ & $0.2000 \mathrm{E}+00$ & $-0.7000 \mathrm{E}+00$ & $0.5000 \mathrm{E}+00$ \\
\hline
\end{tabular}

TABLE 11. The same as Table 5 but for the exact solution of the test number 5 of Balsara (2001) computed with an accuracy of $3 \times 10^{-4}$.

in this direction would be, for instance, the search for an analytic solution for the shock velocity and this will be the subject of future work. Another important problem deserving equal attention is that of the uniqueness of the solution. While a global consensus on this issue still needs to be reached, it will remain essential in order to construct a complete and consistent picture of the exact solution of the Riemann problem in relativistic MHD.

The numerical codes computing the exact solution both when $B^{x}=0$ and when $B^{x} \neq 0$ are available from the authors upon request. Users of the codes can give credit by mentioning the source and citing this paper.

It is a pleasure to thank José Mํa . Martí, José A. Pons and Olindo Zanotti for useful discussions and comments. 


\begin{tabular}{|c|c|c|c|c|c|c|c|}
\hline & $\rho$ & $p$ & $v^{x}$ & $v^{y}$ & $v^{z}$ & $B^{y}$ & $B^{z}$ \\
\hline R1 & $0.1000 \mathrm{E}+01$ & $0.2376 \mathrm{E}+02$ & $0.0000 \mathrm{E}+00$ & $0.3000 \mathrm{E}+00$ & $0.4000 \mathrm{E}+00$ & $0.6000 \mathrm{E}+01$ & $0.2000 \mathrm{E}+01$ \\
R2 & $0.9219 \mathrm{E}+00$ & $0.2083 \mathrm{E}+02$ & $0.6232 \mathrm{E}-01$ & $0.3050 \mathrm{E}+00$ & $0.4193 \mathrm{E}+00$ & $0.5622 \mathrm{E}+01$ & $0.1892 \mathrm{E}+01$ \\
R3 & $0.9219 \mathrm{E}+00$ & $0.2083 \mathrm{E}+02$ & $0.7109 \mathrm{E}-01$ & $0.3669 \mathrm{E}+00$ & $0.2429 \mathrm{E}+00$ & $0.5691 \mathrm{E}+01$ & $0.8502 \mathrm{E}+00$ \\
R4 & $0.1263 \mathrm{E}+01$ & $0.2087 \mathrm{E}+02$ & $0.3886 \mathrm{E}-01$ & $0.1147 \mathrm{E}+00$ & $0.2054 \mathrm{E}+00$ & $0.5130 \mathrm{E}+01$ & $0.7680 \mathrm{E}+00$ \\
R5 & $0.1099 \mathrm{E}+01$ & $0.2087 \mathrm{E}+02$ & $0.3886 \mathrm{E}-01$ & $0.1147 \mathrm{E}+00$ & $0.2054 \mathrm{E}+00$ & $0.5130 \mathrm{E}+01$ & $0.7680 \mathrm{E}+00$ \\
R6 & $0.9130 \mathrm{E}+00$ & $0.2085 \mathrm{E}+02$ & $0.1607 \mathrm{E}-01$ & $-0.5009 \mathrm{E}-01$ & $0.1813 \mathrm{E}+00$ & $0.5505 \mathrm{E}+01$ & $0.8195 \mathrm{E}+00$ \\
R7 & $0.9130 \mathrm{E}+00$ & $0.2085 \mathrm{E}+02$ & $0.1341 \mathrm{E}-01$ & $-0.6599 \mathrm{E}-03$ & $-0.2640 \mathrm{E}-03$ & $0.5073 \mathrm{E}+01$ & $0.2029 \mathrm{E}+01$ \\
R8 & $0.9000 \mathrm{E}+00$ & $0.2030 \mathrm{E}+02$ & $0.0000 \mathrm{E}+00$ & $0.0000 \mathrm{E}+00$ & $0.0000 \mathrm{E}+00$ & $0.5000 \mathrm{E}+01$ & $0.2000 \mathrm{E}+01$ \\
\hline
\end{tabular}

TABLE 12. The same as Table 5 but for the exact solution of the generic Alfvèn test computed with an accuracy of $10^{-10}$.

\section{Appendix A.}

We here report the expressions for the tangential components of the velocity behind the shock (i.e. $v_{b}^{y}, v_{b}^{z}$ ) when expressed as function of post-shock $p$ and $J$. First, we consider $v_{b}^{y}$ as function of $p_{b}, J$ and $v_{b}^{z}$.

$$
\begin{aligned}
v_{b}^{y}= & -\frac{1}{N_{y}}\left\{J ^ { 3 } W _ { a } ^ { 4 } \left[v_{a}^{y}\left(D_{a}+p_{a}+\tau_{a}-\eta_{a}^{2} W_{a}^{2}\right)\left(D_{a}+p_{b}+\tau_{a}-B_{x}^{2}-\eta_{a}^{2} W_{a}^{2}\right)+\right.\right. \\
& B_{a}^{y}\left(B_{x}\left(p_{a}+\tau_{a}\right) v_{a}^{x}+B_{a}^{z}\left(p_{b}+\tau_{a}\right) v_{b}^{z}-\eta_{a}\left(p_{b}+\tau_{a}\right)-\right. \\
& \left.\left.D_{a}\left(\eta_{a}-B_{x} v_{a}^{x}-B_{a}^{z} v_{b}^{z}\right)+\eta_{a}^{3} W_{a}^{2}-\eta_{a}^{2}\left(B_{x} v_{a}^{x}+B_{a}^{z} v_{b}^{z}\right) W_{a}^{2}\right)\right]- \\
& J^{2} D_{a} W_{a}^{2}\left[\left(2 B_{x}^{3} \eta_{a} v_{a}^{y}+B_{x}^{2} v_{a}^{x}\left[\left(D_{a}+p_{a}+\tau_{a}\right) v_{a}^{y}-2 B_{a}^{y} \eta_{a}\right]+\right.\right. \\
& v_{a}^{x}\left(p_{a}-p_{b}\right)\left[2\left(D_{a}+p_{a}+\tau_{a}\right) v_{a}^{y}+B_{a}^{y}\left(B_{a}^{z} v_{b}^{z}-2 \eta_{a}\right)\right]-B_{x}\left\{\eta_{a}\left(3 D_{a}+2 p_{a}+p_{b}+3 \tau_{a}\right) v_{a}^{y}-\right. \\
& \left.\left.B_{a}^{z}\left(p_{a}-p_{b}\right) v_{a}^{y} v_{b}^{z}+B_{a}^{y}\left[\left(p_{a}+\tau_{a}\right)\left(v_{a}^{x 2}-1\right)-3 \eta_{a}^{2}+2 B_{a}^{z} \eta_{a} v_{b}^{z}+\left(p_{b}+\tau_{a}\right) v_{a}^{z} v_{b}^{z}-M D_{a}\right]\right\}\right) W_{a}^{2}- \\
& \left.B_{x} B_{a}^{y}\left(D_{a}+p_{b}+\tau_{a}\right)+\eta_{a}^{2}\left[2\left(p_{b}-p_{a}\right) v_{a}^{x} v_{a}^{y}-B_{x}^{2} v_{a}^{x} v_{a}^{y}+B_{x}\left(3 \eta_{a} v_{a}^{y}-M B_{a}^{y}\right)\right] W_{a}^{4}\right] W_{s}- \\
& J D_{a}^{2} W_{a}^{2}\left[B_{x}^{3} v_{a}^{x}\left(B_{a}^{y}+2 \eta_{a} v_{a}^{y} W_{a}^{2}\right)-B_{x}^{4} v_{a}^{y}+\right. \\
& B_{x} v_{a}^{x}\left(p_{a}-p_{b}\right)\left[2 B_{a}^{y}+\left(2 \eta_{a} v_{a}^{y}+B_{a}^{z} v_{a}^{y} v_{b}^{z}+B_{a}^{y} v_{a}^{z} v_{b}^{z}\right) W_{a}^{2}\right]+ \\
& Q\left[\left(D_{a}+p_{a}+\tau_{a}\right) v_{a}^{y}-\eta_{a}\left(B_{a}^{y}+\eta_{a} v_{a}^{y} W_{a}^{2}\right)\right]+ \\
& \left.B_{x}^{2}\left(B_{a}^{y}\left(B_{a}^{z} v_{b}^{z}-3 \eta_{a}+2 M \eta_{a} W_{a}^{2}\right)+v_{a}^{y}\left[D_{a}+p_{a}+\tau_{a}-\left(3 \eta_{a}^{2}+\left(p_{b}-p_{a}\right) v_{a}^{z} v_{b}^{z}\right) W_{a}^{2}\right]\right)\right] W_{s}^{2}+ \\
& B_{x} D_{a}^{3}\left(B_{x}^{3} v_{a}^{x} v_{a}^{y} W_{a}^{2}-B_{x}\left(p_{a}-p_{b}\right) v_{a}^{x} v_{a}^{y} v_{a}^{z} v_{b}^{z} W_{a}^{4}-Q\left(B_{a}^{y}+\eta_{a} v_{a}^{y} W_{a}^{2}\right)-\right. \\
& \left.\left.B_{x}^{2}\left[B_{a}^{y}+\left(\eta_{a} v_{a}^{y}-M B_{a}^{y}\right) W_{a}^{2}\right]\right) W_{s}^{3}\right\}
\end{aligned}
$$


where we have defined $M \equiv\left(1-v_{a}^{x^{2}}-v_{a}^{z} v_{b}^{z}\right), Q \equiv W_{a}^{2}\left(p_{a}-p_{b}\right)\left(v_{a}^{x^{2}}-1\right)$ and

$$
\begin{aligned}
N_{y} \equiv & W_{a}^{2}\left\{B _ { x } D _ { a } W _ { a } ^ { 2 } W _ { s } \left(J ^ { 2 } \left[2 B_{a}^{y 2} \eta_{a}-\right.\right.\right. \\
& \left.3 \eta_{a}\left(D_{a}+p_{b}+\tau_{a}\right)+3 \eta_{a}^{3} W_{a}^{2}+B_{a}^{y} v_{a}^{y}\left(D_{a}-p_{a}+2 p_{b}+\tau_{a}-\eta_{a}^{2} W_{a}^{2}\right)\right]+ \\
& \left.2 D_{a} J\left(p_{a}-p_{b}\right) v_{a}^{x}\left(2 \eta_{a}-B_{a}^{y} v_{a}^{y}\right) W_{s}+D_{a}^{2} \eta_{a}\left(p_{a}-p_{b}\right)\left(v_{a}^{x 2}-1\right) W_{s}^{2}\right)- \\
& B_{x}^{4} D_{a}^{2} W_{s}^{2}\left(J+D_{a} v_{a}^{x} W_{s}\right)-W_{a}^{2}\left[J\left(D_{a}+p_{b}+\tau_{a}-\eta_{a}^{2} W_{a}^{2}\right)+\right. \\
& \left.D_{a}\left(p_{b}-p_{a}\right) v_{a}^{x} W_{s}\right]\left[J^{2}\left(D_{a}+p_{b}+\tau_{a}-\eta_{a}^{2} W_{a}^{2}\right)+2 D_{a} J\left(p_{b}-p_{a}\right) v_{a}^{x} W_{s}-\right. \\
& \left.B_{a}^{y 2} J^{2}+D_{a}^{2}\left(p_{b}-p_{a}\right)\left(v_{a}^{x 2}-1\right) W_{s}^{2}\right]+B_{x}^{3} D_{a} W_{s}\left[2 \eta_{a} J^{2} W_{a}^{2}+\right. \\
& \left.2 D_{a} \eta_{a} J v_{a}^{x} W_{a}^{2} W_{s}+D_{a}^{2}\left(\eta_{a}-B_{a}^{y} v_{a}^{y}\right) W_{s}^{2}\right]+B_{x}^{2}\left[J^{3} W_{a}^{2}\left(D_{a}+p_{b}+\tau_{a}-\eta_{a}^{2} W_{a}^{2}\right)+\right. \\
& D_{a} J^{2} v_{a}^{x} W_{a}^{2}\left(D_{a}-2 p_{a}+3 p_{b}+\tau_{a}-\eta_{a}^{2} W_{a}^{2}\right) W_{s}+ \\
& D_{a}^{2} J\left(D_{a}+p_{b}-B_{a}^{y 2}+\tau_{a}+\left[2 B_{a}^{y} \eta_{a} v_{a}^{y}-3 \eta_{a}^{2}-\left(p_{a}-p_{b}\right)\left(3 v_{a}^{x 2}-1+v_{a}^{y 2}\right)\right] W_{a}^{2}\right) W_{s}^{2}- \\
& \left.\left.D_{a}^{3} v_{a}^{x} v_{a}^{z 2} W_{a}^{2}\left(p_{b}-p_{a}\right) W_{s}^{3}\right]\right\} .
\end{aligned}
$$

We next consider the expression of $v_{b}^{z}$ as function of post-shock $p$ and $J$

$$
\begin{aligned}
v_{b}^{z}= & -\frac{1}{N_{z}}\left\{J ^ { 3 } W _ { a } ^ { 4 } \left[v _ { a } ^ { z } ( D _ { a } + p _ { a } + \tau _ { a } - \eta _ { a } ^ { 2 } W _ { a } ^ { 2 } ) \left(D_{a}-B^{x 2}-B_{a}^{y 2}+\right.\right.\right. \\
& \left.p_{b}+\tau_{a}-\eta_{a}^{2} W_{a}^{2}\right)+B_{a}^{z}\left(\left(p_{a}+\tau_{a}\right)\left(B^{x} v_{a}^{x}+B_{a}^{y} v_{a}^{y}\right)-\eta_{a}\left(p_{b}+\tau_{a}\right)+\right. \\
& \left.\left.D_{a}\left(B^{x} v_{a}^{x}+B_{a}^{y} v_{a}^{y}-\eta_{a}\right)+\eta_{a}^{3} W_{a}^{2}-\eta_{a}^{2}\left(B^{x} v_{a}^{x}+B_{a}^{y} v_{a}^{y}\right) W_{a}^{2}\right)\right]+ \\
& D_{a} J^{2} W_{a}^{2}\left[B^{x} B_{a}^{z}\left(D_{a}+p_{b}+\tau_{a}\right)+\left(B ^ { x } B _ { a } ^ { z } \left[2 B_{a}^{y} \eta_{a} v_{a}^{y}-3 \eta_{a}^{2}+\right.\right.\right. \\
& \left.D_{a}\left(v_{a}^{x 2}+v_{a}^{y 2}-1\right)+\left(p_{a}+\tau_{a}\right)\left(v_{a}^{x 2}+v_{a}^{y 2}-1\right)\right]- \\
& 2 B^{x 3} \eta_{a} v_{a}^{z}-B^{x}\left[2 B_{a}^{y 2} \eta_{a}-\eta_{a}\left(3 D_{a}+2 p_{a}+p_{b}+3 \tau_{a}\right)+\right. \\
& B_{a}^{y}\left(D_{a}+p_{a}+\tau_{a}\right) v_{a}^{y} v_{a}^{z}-B^{x 2} v_{a}^{x}\left[\left(D_{a}+p_{a}+\tau_{a}\right) v_{a}^{z}-2 B_{a}^{z} \eta_{a}\right]- \\
& \left.2\left(p_{a}-p_{b}\right) v_{a}^{x}\left[\left(D_{a}+p_{a}+\tau_{a}\right) v_{a}^{z}-B_{a}^{z} \eta_{a}\right]\right) W_{a}^{2}+ \\
& \left.\eta_{a}^{2}\left(B^{x} B_{a}^{z}\left(v_{a}^{x 2}+v_{a}^{y 2}-1\right)-\left(B^{x 2} v_{a}^{x}-3 B^{x} \eta_{a}+2 p_{a} v_{a}^{x}-2 p_{b} v_{a}^{x}+B^{x} B_{a}^{y} v_{a}^{y}\right) v_{a}^{z}\right) W_{a}^{4}\right] W_{s}- \\
& D_{a}^{2} J W_{a}^{2}\left(2 B^{x}\left(p_{a}-p_{b}\right) v_{a}^{x}\left(B_{a}^{z}+\eta_{a} v_{a}^{z} W_{a}^{2}\right)-B^{x 4} v_{a}^{z}+B^{x 3} v_{a}^{x}\left(B_{a}^{z}+2 \eta_{a} v_{a}^{z} W_{a}^{2}\right)+\right. \\
& B^{x 2}\left\{v_{a}^{z}\left[D_{a}-B_{a}^{y 2}+p_{a}+\tau_{a}-\eta_{a}\left(3 \eta_{a}-2 B_{a}^{y} v_{a}^{y}\right) W_{a}^{2}\right]+\right. \\
& \left.B_{a}^{z}\left[B_{a}^{y} v_{a}^{y}-3 \eta_{a}-2 \eta_{a}\left(v_{a}^{x 2}+v_{a}^{y 2}-1\right) W_{a}^{2}\right]\right\}+ \\
& \left.\left(p_{a}-p_{b}\right)\left(v_{a}^{x 2}-1\right) W_{a}^{2}\left[\left(D_{a}+p_{a}+\tau_{a}\right) v_{a}^{z}-\eta_{a}\left(B_{a}^{z}+\eta_{a} v_{a}^{z} W_{a}^{2}\right)\right]\right) W_{s}^{2}+ \\
& B^{x} D_{a}^{3}\left[B_{x}^{3} v_{a}^{x} v_{a}^{z} W_{a}^{2}-\left(p_{a}-p_{b}\right)\left(v_{a}^{x 2}-1\right) W_{a}^{2}\left(B_{a}^{z}+\eta_{a} v_{a}^{z} W_{a}^{2}\right)-\right. \\
& \left.\left.B_{x}^{2}\left(B_{a}^{z}+\left[B_{a}^{z}\left(v_{a}^{x 2}+v_{a}^{y 2}-1\right)+\left(\eta_{a}-B_{a}^{y} v_{a}^{y}\right) v_{a}^{z}\right] W_{a}^{2}\right)\right] W_{s}^{3}\right\} \\
&
\end{aligned}
$$


where

$$
\begin{aligned}
N_{z} \equiv & W_{a}^{2}\left\{B ^ { x } D _ { a } W _ { a } ^ { 2 } W _ { s } \left\{J ^ { 2 } \left[2 B_{a}^{y 2} \eta_{a}+2 B_{a}^{z 2} \eta_{a}-3 \eta_{a}\left(D_{a}+p_{b}+\tau_{a}\right)+3 \eta_{a}^{3} W_{a}^{2}+\right.\right.\right. \\
& \left.B_{a}^{y} v_{a}^{y}\left(D_{a}-p_{a}+2 p_{b}+\tau_{a}-\eta_{a}^{2} W_{a}^{2}\right)+B_{a}^{z} v_{a}^{z}\left(D_{a}-p_{a}+2 p_{b}+\tau_{a}-\eta_{a}^{2} W_{a}^{2}\right)\right]+ \\
& \left.2 D_{a} J\left(p_{a}-p_{b}\right) v_{a}^{x}\left(2 \eta_{a}-B_{a}^{y} v_{a}^{y}-B_{a}^{z} v_{a}^{z}\right) W_{s}+D_{a}^{2} \eta_{a}\left(p_{a}-p_{b}\right)\left(v_{a}^{x 2}-1\right) W_{s}^{2}\right\}- \\
& B_{x}^{4} D_{a}^{2} W_{s}^{2}\left(J+D_{a} v_{a}^{x} W_{s}\right)-W_{a}^{2}\left[J\left(D_{a}+p_{b}+\tau_{a}-\eta_{a}^{2} W_{a}^{2}\right)-\right. \\
& \left.D_{a}\left(p_{a}-p_{b}\right) v_{a}^{x} W_{s}\right]\left[J^{2}\left(D_{a}-B_{a}^{y 2}-B_{a}^{z 2}+p_{b}+\tau_{a}-\eta_{a}^{2} W_{a}^{2}\right)-\right. \\
& \left.2 D_{a} J\left(p_{a}-p_{b}\right) v_{a}^{x} W_{s}-D_{a}^{2}\left(p_{a}-p_{b}\right)\left(v_{a}^{x 2}-1\right) W_{s}^{2}\right]+ \\
& B_{x}^{3} D_{a} W_{s}\left[2 \eta_{a} J^{2} W_{a}^{2}+2 D_{a} \eta_{a} J v_{a}^{x} W_{a}^{2} W_{s}+D_{a}^{2}\left(\eta_{a}-B_{a}^{y} v_{a}^{y}-B_{a}^{z} v_{a}^{z}\right) W_{s}^{2}\right]+ \\
& B_{x}^{2}\left(J^{3} W_{a}^{2}\left(D_{a}+p_{b}+\tau_{a}-\eta_{a}^{2} W_{a}^{2}\right)+D_{a} J^{2} v_{a}^{x} W_{a}^{2}\left(D_{a}-2 p_{a}+3 p_{b}+\tau_{a}-\eta_{a}^{2} W_{a}^{2}\right) W_{s}+\right. \\
& D_{a}^{2} J\left\{D_{a}-B_{a}^{y 2}-B_{a}^{z 2}+p_{b}+\tau_{a}+\left[2 \eta_{a}\left(B_{a}^{y} v_{a}^{y}+B_{a}^{z} v_{a}^{z}\right)-3 \eta_{a}^{2}-\right.\right. \\
& \left.\left.\left.\left.\left(p_{a}-p_{b}\right)\left(3 v_{a}^{x 2}+v_{a}^{y 2}+v_{a}^{z 2}-1\right)\right] W_{a}^{2}\right\} W_{s}^{2}\right)\right\}
\end{aligned}
$$

\section{Appendix B.}

The explicit form for the system of ODEs to be solved numerically to determine the solution across a rarefaction wave within the $p$-method is given by the following set of equations in which the total pressure $p$ plays the role of the self-similar variable

$$
\begin{aligned}
\frac{\mathrm{d} \rho}{\mathrm{d} p}= & -\rho\left(W^{2} v_{x}+\frac{1}{v_{x}-\xi}\right) \frac{\mathrm{d} v_{x}}{\mathrm{~d} p}-\rho W^{2} v_{y} \frac{\mathrm{d} v_{y}}{\mathrm{~d} p}-\rho W^{2} v_{z} \frac{\mathrm{d} v_{z}}{\mathrm{~d} p} \\
\frac{\mathrm{d} v_{x}}{\mathrm{~d} p}= & R\left\{\left(\rho h_{g} W^{2}+B_{x}^{2}\right)\left(\xi-v_{x}\right)\left(v_{x} \xi-1\right)+B_{x}^{2} \frac{\xi v_{x}-1}{W^{2}\left(v_{x}-\xi\right)}+B_{x}^{2} \xi\left(v_{y}^{2}+v_{z}^{2}\right)+\right. \\
& \left.B_{x}\left[\eta\left(\xi^{2}-1\right)-B_{x} v_{x}\left(1-2 v_{x} \xi+\xi^{2}\right)\right]\right\} \\
\frac{\mathrm{d} v_{y}}{\mathrm{~d} p}= & R\left\{2 B_{x} v_{y}\left(\eta-B_{z} v_{z}\right) \xi-B_{x}^{2} v_{y} \xi\left(\xi+v_{x}\right)+\right. \\
& v_{y}\left[B_{z}^{2}+W^{2}\left(\eta^{2}-w\right)\right]\left(v_{x}-\xi\right) \xi+B_{y}^{2} v_{y}\left(v_{x} \xi-1\right)+B_{y} B_{z} v_{z}\left(\xi^{2}-1\right)+ \\
& \left.B_{x} B_{y} \frac{\left(v_{y}^{2}+v_{z}^{2}-1\right)+\left(v_{x}-2 v_{x} v_{y}^{2}\right) \xi+\left(1+v_{y}^{2}-v_{z}^{2}\right) \xi^{2}-v_{x} \xi^{3}}{\left(v_{x}-\xi\right)}\right\} \\
\frac{\mathrm{d} v_{z}}{\mathrm{~d} p}= & R\left\{2 B_{x}\left(\eta-B_{y} v_{y}\right) v_{z} \xi-B_{x}^{2} v_{z} \xi\left(v_{x}+\xi\right)+\right. \\
& v_{z}\left[B_{y}^{2}+W^{2}\left(\eta^{2}-w\right)\right]\left(v_{x}-\xi\right) \xi+B_{y} B_{z} v_{y}\left(\xi^{2}-1\right)+v_{z} B_{z}^{2}\left(v_{x} \xi-1\right)+ \\
& \left.B_{x} B_{z} \frac{\left(v_{y}^{2}+v_{z}^{2}-1\right)+\left(v_{x}-2 v_{x} v_{z}^{2}\right) \xi+\left(1-v_{y}^{2}+v_{z}^{2}\right) \xi^{2}-v_{x} \xi^{3}}{\left(v_{x}-\xi\right)}\right\} \\
\frac{\mathrm{d} B_{y}}{\mathrm{~d} p}= & -\frac{W^{2}\left(B_{y}-B_{y} v_{x} \xi+B_{x} v_{y} \xi\right)}{B_{x}^{2}+2 B_{x} \eta W^{2}\left(v_{x}-\xi\right)+W^{4}\left(\eta^{2}-w\right)\left(v_{x}-\xi\right)^{2}} \\
\frac{\mathrm{d} B_{z}}{\mathrm{~d} p}= & -\frac{W^{2}\left(B_{z}-B_{z} v_{x} \xi+B_{x} v_{z} \xi\right)}{B_{x}^{2}+2 B_{x} \eta W^{2}\left(v_{x}-\xi\right)+W^{4}\left(\eta^{2}-w\right)\left(v_{x}-\xi\right)^{2}},
\end{aligned}
$$


where we have defined

$$
R \equiv \frac{1}{\rho h_{g} W^{4}\left(\eta^{2}-w\right)\left(V_{A}^{+}-\xi\right)\left(V_{A}^{-}-\xi\right)},
$$

with

$$
V_{A}^{ \pm} \equiv v_{x}+\frac{B_{x}}{W^{2}(\eta \pm \sqrt{w})},
$$

being the Alfvèn velocities in the two directions. Note that the set of ODEs has a singular point if the characteristic velocity of the slow or fast magnetosonic waves is equal to the Alfven velocity [cf. eq. [B 7] ] and cannot be solved in this case without a proper regularization. This procedure is not included in the numerical code made available upon request.

\section{REFERENCES}

Aloy, M. A., IbÁÑez, J. M., Martí, J. M. AND MÜller, E. 1999, GENESIS: A High-Resolution Code for Three-dimensional Relativistic Hydrodynamics, ApJS, 122, 151.

Anile, A. M. 1989, Relativistic Fluids and Magneto-Fluids, Cambridge University Press.

Antón, L., Zanotti, L., Miralles, J. A., Martí, J. M., Ibáñez, J. M프., Pons, J. A., And Font, J. A. 2006, Numerical $3+1$ General Relativistic Magnetohydrodynamics: A local characteristic approach, ApJ, 637, 296.

Baiotti, L. Hawke I., Montero, P., Loeffler F., Rezzolla L., Stergioulas N., Font, J. A. AND SEIDEL, E. 2005, Three-dimensional relativistic simulations of rotating neutron star collapse to a Kerr black hole, Phys. Rev. D, 71024035.

BAlsara, D. 2001, Total Variation Diminishing Scheme for Relativistic Magnetohydrodynamics, ApJS, 132, 83.

BRIO, M. AND WU, C.-C. 1988, An upwind differencing scheme for the equations of ideal magnetohydrodynamics, J. Comput. Phys., 75, 400.

DaI, W. And Woodward, P. R. 1994, An Approximate Riemann Solver for Ideal Magnetohydrodynamics, J. Comput. Phys., 111, 354.

Del Zanna, L., BUCCIANTINI, N. AND LondRillo, P. 2003, An efficient shock-capturing central-type scheme for multidimensional relativistic flows. II. Magnetohydrodynamics, $A \& A, \mathbf{4 0 0}, 397$.

De Villiers, J.-P. And Hawley, J. F. 2003, A Numerical Method for General Relativistic Magnetohydrodynamics, ApJ, 589, 458.

Duez, M. D., Shapiro, S. L. ANd Yo, H. J., 2004, Relativistic Hydrodynamic Evolutions with Black Hole Excision, Phys. Rev. D., 69104106

Duez, M. D., LiU, Y. T., Shapiro, S. L. And Stephens, B. C. 2005, Relativistic Magnetohydrodynamics In Dynamical Spacetimes: Numerical Methods And Tests, Phys. Rev. D., 72, 024028.

EInfEldt, B. 1988, On Godunov-Type Methods for Gas Dynamics, SIAM J. Numer. Anal., 25(2), 294.

Falle, S. A. E. G., Komissarov, S. S. And Joarder, P. 1998, A multidimensional upwind scheme for magnetohydrodynamics, MNRAS, 297, 265.

FAlle, S. A. E. G. AND Komissarov, S. S. 2001, On the inadmissibility of non-evolutionary shocks, J. Plasma Physics, 65, 29.

Font, J. A. 2003 Numerical Hydrodynamics in General Relativity, Living Reviews in Relativity, Vol 6, 4.

FOnT, J. A., DAIGne, F., 2002, The runaway instability of thick discs around black holes - I. The constant angular momentum case, MNRAS, 334, 383.

Fragile, P. C. 2005, Quasi-Periodic Oscillations in Relativistic Tori, Proceedings of the 22nd Texas Meeting on Relativistic Astrophysics, Stanford University, astro-ph/0503305

Gammie, C. F., McKinney, J. C. And Tóth, G. 2003, HARM: A Numerical Scheme for General Relativistic Magnetohydrodynamics, ApJ, 589, 444.

Godunov, S. K. 1959, A Finite Difference Method for the Numerical Computation and Discontinuous Solutions of the Equations of Fluid Dynamics, Mat. Sb., 47, 271.

Harten, A., LAX, P. D., VAn LeER, B. 1983, On Upstream Differencing and Godunov-Type Schemes for Hyperbolic Conservation Laws, SIAM Rev., 25(1), 35.

JefFrey, A., TAniUti, T. 1964, Non-linear wave propagation, Academic Press, New York.

JEFFREY, A. 1966, Magnetohydrodynamics, Oliver \& Boyd. 
Komissarov, S.S. 1997, On the properties of Alfvén waves in relativistic magnetohydrodynamics, Phys. Lett. A, 232, 435.

Komissarov, S.S. 1999, A Godunov-type scheme for relativistic magnetohydrodynamics, MNRAS, 303, 343.

KOMISSAROV, S.S. 2003, Limit shocks of relativistic magnetohydrodynamics, MNRAS, 341, 717.

KOMISSAROV, S.S. 2004, General relativistic magnetohydrodynamics simulations of monopole magnetospheres of black holes, MNRAS, 350, 1431.

Landau, L. D. And Lifshitz, E. M. 1984, Electrodynamics of Continuous Media in Course of Theoretical Physics, Pergamon Press Ltd.

LEVEQue, R. J. 1992, Numerical Methods for Conservation Laws (2nd. Edition), Birkhäuser.

LiCHNEROWICZ, A. 1967, Relativistic Hydrodynamics and Magnetohydrodynamics, W. A. Benjamin INC.

MARTí, J. M. AND MÜLlER, E. 1994, The analytical solution of the Riemann problem in relativistic hydrodynamics, J. Fluid. Mech., 258, 317.

Martí, J.M. \& Müller, E. 2003 Numerical Hydrodynamics in Special Relativity, Living Reviews in Relativity, Vol. 6, 7.

Mizuno, Y., Yamada, S., Koide, S. And Shibata, K. 2004, General Relativistic Magnetohydrodynamic Simulations of Collapsars: Rotating Black Hole Cases, ApJ, 606, 395.

Myong, R. S., RoE, P. L., 1997A, Shock Waves and Rarefaction Waves in Magnetohydrodynamics. Part 1. A Model System, J. Plasma Phys., 58, 485.

Myong, R. S., RoE, P. L., 1997B, Shock Waves and Rarefaction Waves in Magnetohydrodynamics. Part 2. The MHD System, J. Plasma Phys., 58, 521.

Pons, J. A., Martí, J. M. AND MüLler, E. 2000, The exact solution of the Riemann problem with non-zero tangential velocities in relativistic hydrodynamics, J. Fluid. Mech., 422, 125.

Rezzolla, L. AND ZanotTi, O. 2001, An improved exact Riemann solver for relativistic hydrodynamics, J. Fluid. Mech., 449, 395.

Rezzolla, L. And ZanotTi, O. 2002, New Relativistic Effects in the Dynamics of Nonlinear Hydrodynamical Waves, Phys. Rev. Lett., 89, 11.

Rezzolla, L., ZanotTi, O. AND Pons, J. A. 2003, An improved exact Riemann solver for multidimensional relativistic flows, J. Fluid. Mech., 479, 199.

Romero, R., Martí, J. M., Pons, J. A., Ibáñez, J. M프. And Miralles, J. A. 2005, The exact solution of the Riemann problem in relativistic MHD with tangential magnetic fields, J. Fluid. Mech., in press

RYU, D. And Jones, T. W. 1995, Numerical Magnetohydrodynamics in Astrophysics: Algorithm and Tests for One-Dimensional Flow, ApJ, 442, 228.

Shibata, M,. AND SeKiguchi, Y. 2005, Three-dimensional simulations of stellar core collapse in full general relativity: Nonaxisymmetric dynamical instabilities, Phys. Rev. D, 71024014.

Taub, A. H. 1948, Relativistic Rankine-Hugoniot Equations, Phys. Rev., 74, 328.

TAub, A. H. 1978, Relativistic Fluid Mechanics, Ann. Rev. Fluid. Mech., 10, 301.

Toro, E. F. 1999, Riemann Solvers and Numerical Methods for Fluid Dynamics, 2nd edition, SpringerVerlag.

TORRILHON, M. 2003A, Non-uniform convergence of finite volume schemes for Riemann problems of ideal magnetohydrodynamics, J. Comput. Phys., 192, 73.

TORRILHON, M. 2003B, Uniqueness conditions for Riemann problems of ideal magnetohydrodynamics, J. Plasma Physics, 69, 253.

TORRILHON, M. AND BALSARA, D. S. 2004, High order WENO schemes: investigations on non-uniform convergence for MHD Riemann problems, J. Comput. Phys., 201, 586.

van Putten, M. H. P. M. 1993, A Numerical Implementation of MHD in Divergence Form, J. Comput. Phys., 105, 339.

Zanotti, O., ReZzolla, L., Font, J. A., 2003, Quasi-periodic accretion and gravitational waves from oscillating "toroidal neutron stars", MNRAS, 341, 832. 